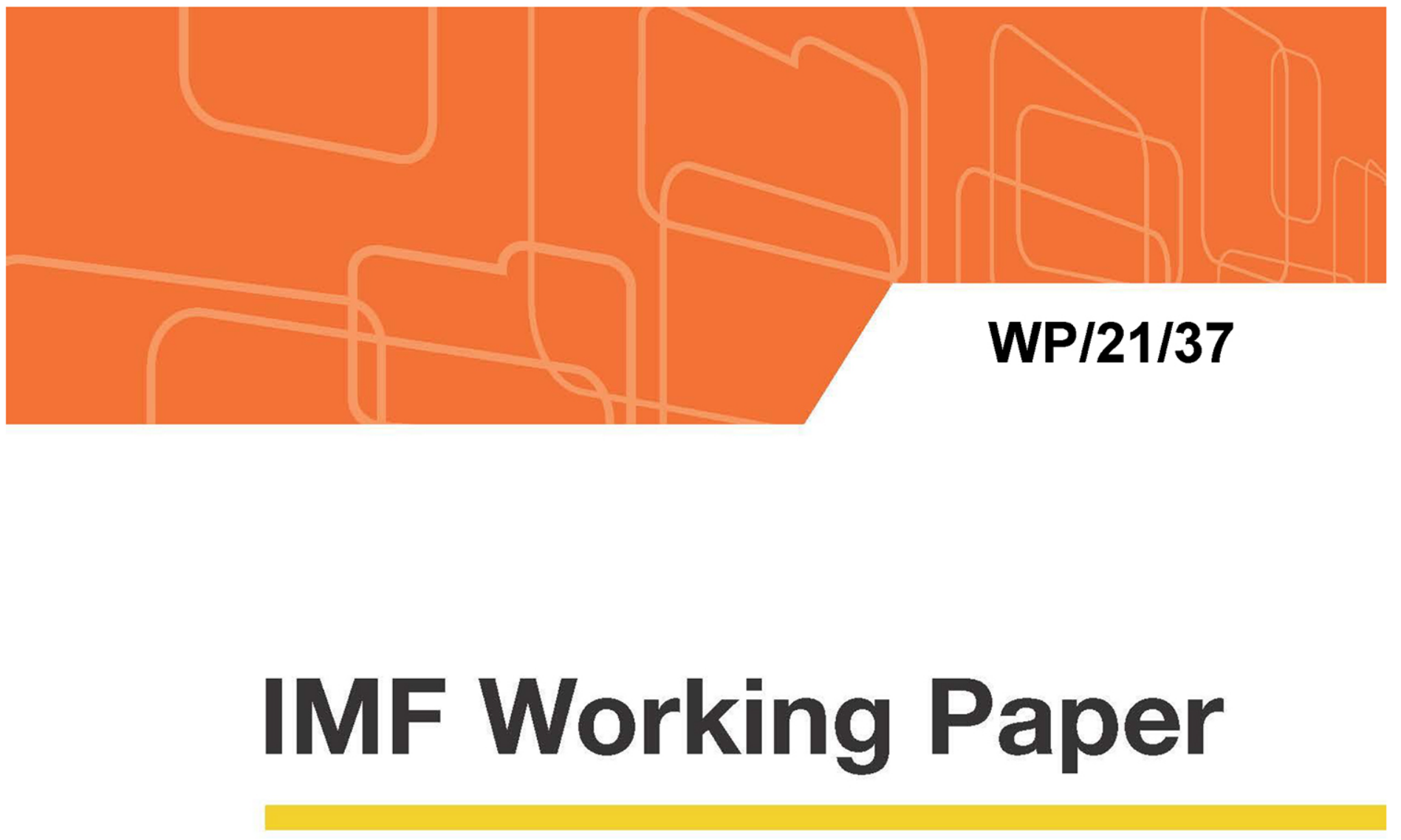

\title{
A Unified Model of Cohort Mortality for Economic Analysis
}

by Adriana Lleras-Muney and Flavien Moreau

IMF Working Papers describe research in progress by the author(s) and are published to elicit comments and to encourage debate. The views expressed in IMF Working Papers are those of the author(s) and do not necessarily represent the views of the IMF, its Executive Board, or IMF management. 


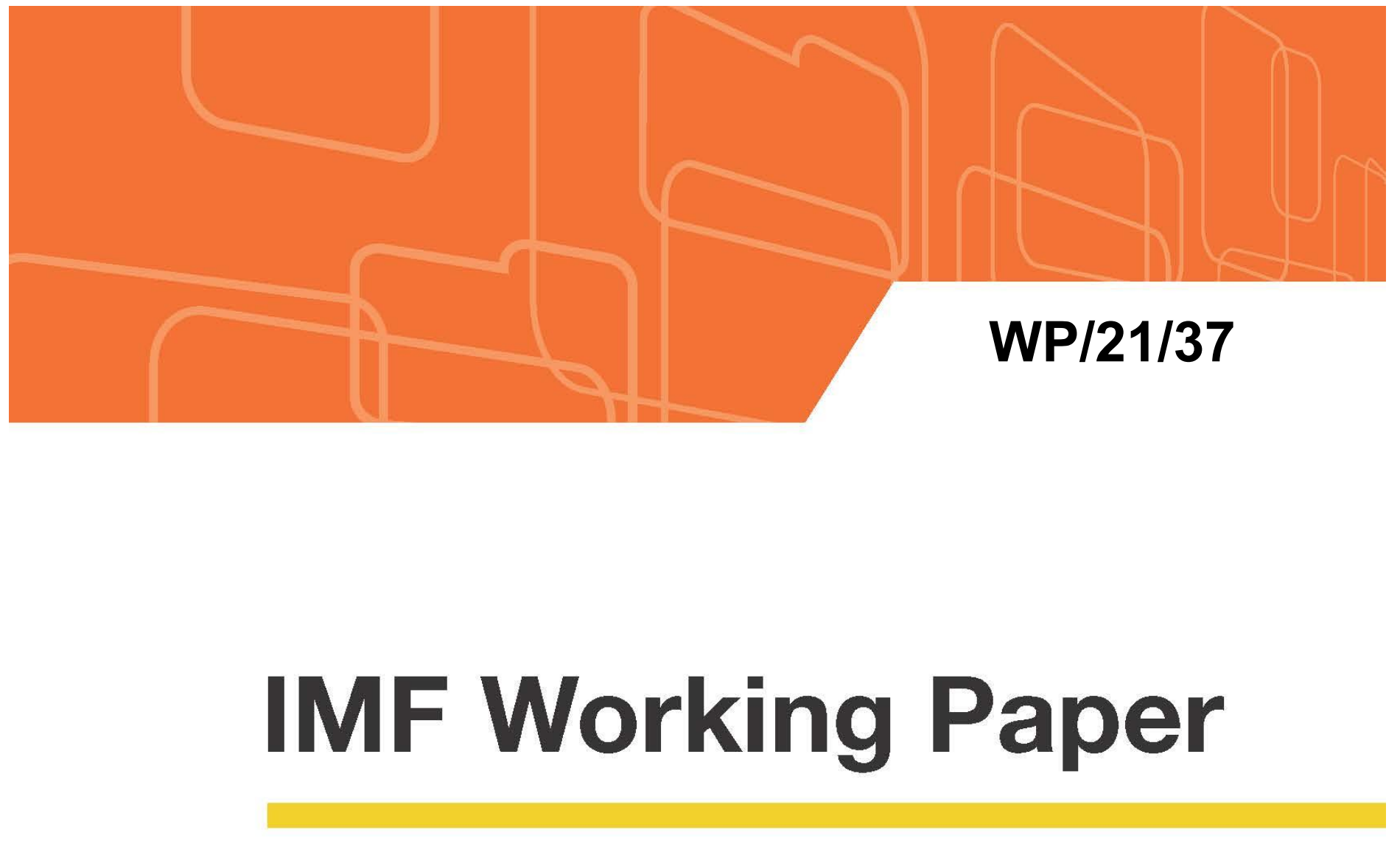

\section{A Unified Model of Cohort Mortality for Economic Analysis}

by Adriana Lleras-Muney and Flavien Moreau

IMF Working Papers describe research in progress by the author(s) and are published to elicit comments and to encourage debate. The views expressed in IMF Working Papers are those of the author(s) and do not necessarily represent the views of the IMF, its Executive Board, or IMF management.

I N T E R N A T I O N A L M O N E T A R Y F U N D 


\title{
IMF Working Paper
}

\author{
African Department
}

\section{A Unified Model of Cohort Mortality for Economic Analysis \\ Prepared by Adriana Lleras-Muney and Flavien Moreau}

Authorized for distribution by Amine Mati

February 2021

\section{IMF Working Papers describe research in progress by the author(s) and are published to elicit comments and to encourage debate. The views expressed in IMF Working Papers are those of the author(s) and do not necessarily represent the views of the IMF, its Executive Board, or IMF management.}

\begin{abstract}
We propose a dynamic production function of population health and mortality from birth onwards. Our parsimonious model provides an excellent fit for the mortality and survival curves for both primate and human populations since 1816. The model sheds light on the dynamics behind many phenomena documented in the literature, including (i) the existence and evolution of mortality gradients across socio-economic statuses, (ii) non-monotonic dynamic effects of in-utero shocks, (iii) persistent or "scarring" effects of wars and (iv) mortality displacement after large temporary shocks such as extreme weather.
\end{abstract}

JEL Classification Numbers: I10, J11.

Keywords: Mortality, Health, In-utero shocks, Selection, Scarring Author's E-Mail Address: fmoreau@imf.org 


\title{
A Unified Model of Cohort Mortality for Economic Analysis
}

\author{
Adriana Lleras-Muney and Flavien Moreau*
}

November 2, 2020

\begin{abstract}
We propose a dynamic production function of population health and mortality from birth onwards. Our parsimonious model provides an excellent fit for the mortality and survival curves for both primate and human populations since 1816. The model sheds light on the dynamics behind many phenomena documented in the literature, including (i) the existence and evolution of mortality gradients across socio-economic statuses, (ii) non-monotonic dynamic effects of in-utero shocks, (iii) persistent or "scarring" effects of wars and (iv) mortality displacement after large temporary shocks such as extreme weather.

JEL: I10, J11
\end{abstract}

Keywords: Mortality, Health, In-utero shocks, Selection, Scarring.

\footnotetext{
*Corresponding author: Adriana Lleras-Muney, Economics Department, UCLA; email: alleras@econ.ucla.edu. Flavien Moreau, International Monetary Fund; email: flavienm@gmail.com. The views expressed in this document are those of the authors and do not necessarily represent those of the IMF or its Executive Board or Management. We are very grateful to David Atkin, Andy Atkeson, David Cutler, Jeff Ely, Price Fishback, Titus Galama, Patrick Heuveline, Bo Honoré and Wei Huang for their advice and to seminar participants at Stanford University, University of British Columbia, University of Connecticut, USC, and UCLA and the NBER Cohort meetings. We are also very grateful to Hualei Shang and Wan Zhang for their outstanding research assistance. This work used computational and storage services associated with the Hoffman2 Shared Cluster provided by UCLA Institute for Digital Research and Education's Research Technology Group. This project was supported by the California Center for Population Research at UCLA (CCPR), which receives core support (P2C- HD041022) from the Eunice Kennedy Shriver National Institute of Child Health and Human Development (NICHD). All errors are our own.
} 
We propose a coherent framework to understand how population health and mortality evolve from birth onwards, and how economic and other environmental factors early in life affect this evolution. Statistical and economic models of health and mortality typically only concentrate on adults. Yet a large literature now documents that events and investments in utero and throughout childhood are powerful predictors of both economic and health outcomes later in life (Almond and Currie 2011, Almond et al. 2018). In the absence of such a quantitative model, it is difficult to predict how shocks will affect population health at various ages, and even harder to design optimal investment or compensation policies (Almond et al., 2018).

We present a simple dynamic model of the production of health from birth to death for a heterogenous population. In the spirit of classic demographic work (Vaupel et al., 1979), the model assumes that some individuals are born more frail than others and tend to die young. Subsequently, the health distribution of the survivors evolves according to a simple law of motion that depends on the level of external resources and their distribution. ${ }^{1}$ As in Grossman (1972), the health stock deteriorates with age but can increase if resources are invested. But unlike Grossman (1972), resources in our model are stochastic, a crucial distinction. In addition, individuals can die from accidents unrelated to their health status. These "external" causes of death play an important role in explaining the level of mortality during the adolescent years.

Our quantitative model can account for several stylized facts documented in the literature. We start by estimating the model separately for more than 100 birth cohorts born in the early 19th century and later, using high quality data from the Human Mortality Database. Despite vast changes in life expectancy throughout the period, the model provides an excellent characterization of the age-profiles of mortality for each of these cohorts. The estimated model is consistent with the following stylized facts: (1) the profile of log mortality rates by age has a J-shape, and (2) survival curves for humans have "rectangularized" over the last two centuries, that is, survival curves have become flatter thoughout life and then drop abruptly at older ages). We then show that simple extensions of this model can be used to rationalize various well-documented mortality phenomena, including

\footnotetext{
${ }^{1}$ The model can be seen as formally similar to the stochastic processes used to model corporate defaults (Lando, 2004).
} 
(3) persistent gaps in log mortality rates across populations with different socioeconomic status that fall with age (aka "SES gradients"); (4) non-monotonic health impacts of in-utero shocks over the lifetime; (5) scarring effects of short-term negative shocks such as wars and (6) harvesting or displacement effects due to environmental shocks such as hot weather.

The evolution of mortality over the lifetime is remarkably similar across human populations and in fact across most primates. Because of this regularity, demographers have searched for a "unified" model of mortality that would predict mortality from birth to death at least since the early 19th century (Gompertz, 1825). Like much of the following literature (e.g. Li and Anderson, 2013) Gompertz's model accounts for mortality only after a certain age, focusing on the roughly log-linear portion of the mortality curve after age 30-40. There are a few exceptions. An early model proposed by Heligman and Pollard (1980) describes the probability of dying at a given age for all ages. More recently Sharrow and Anderson (2016) and Palloni and Beltrán-Sánchez (2016) propose alternative statistical models of survival rates that also fit observed lifetime survival curves well.

The main contribution of this paper is to provide a production function that describes the evolution of a population's health and mortality starting at birth that is suited for tracking the long term impacts of various insults and investments. To that end, our approach differs in one fundamental aspect from the demographic approach. As in the seminal Grossman (1972) model, we model directly how the health stock of each individual evolves, rather than only modeling the mortality or survival rates of the aggregate population. This approach is better suited for studying how various shocks affect the health and mortality of the population overtime - we can easily model inputs into health directly and trace their effects as cohorts age by tracking the evolution of the distribution of health. We demonstrate this by studying the effects of increasing lifetime resources, and the impact of negative in-utero shocks on a population's subsequent average health and mortality. We also study the effects of temporary shocks such as wars or bad weather.

Our basic model is more parsimonious than the original Grossman model, or its most recent successors (Dalgaard and Strulik, 2014 or Galama and Van Kippersluis, 2018). We a focus on a production process only and initially ignore maximzing behavior. Our main innovation relative to these papers is to provide a unified framework for mortality at all ages, including childhood, and to allow for hetero- 
geneous endowments. ${ }^{2}$ Including this key childhood period allows us to match the pattern of declining mortality among children (up to adolescence). Alternative state-of-the art models, such as Dalgaard and Strulik (2014)'s accumulating health deficits model, or Galama and Van Kippersluis (2018)'s theory of socioeconomics status and mortality, start with adults and thus cannot account for this feature of the data. In our model, mortality declines during childhood due to both selection effects and investments, both of which have consequences on adult mortality. By allowing for heterogeneity and tracking the distribution of health, we can account for selection effects due to mortality every period. To our knowledge, there is no other model that can accurately predict the lifetime health and mortality of a population, while also providing a law of motion for health at the individual level.

We end by considering how optimization might affect our findings. We show that a benevolent planner maximizing life expectancy would redistribute resources from middle ages to children and to the elderly. The optimal health investment schedule is increasing with age among the elderly. We also show that, despite this re-allocation, the basic profile of mortality by age remains similar. Our estimates imply that optimization could lead to substantial gains in life expectancy, though lower than what has been observed in the last two centuries. With additional data our model can be easily extended to further account for optimizing behavior.

This paper proceeds as follows. We start by describing the data and the stylized facts that inform our model in section 1 . We then describe the model and its qualitative properties in Section 2. In section 3 we show that the model does an excellent job at matching the mortality profiles of many cohorts, and discuss which parameters in the model help rationalize the large increases in life expectancy we observe throuhout the period. We also show that the model describes the evolution of chimpanzee mortality well. In section 4 we then investigate how the model can be used to understand the effects of permanent (in utero or SES) and temporary (wars and heat waves) changes in the environment. Section 5 presents our optimization results, and section 6 concludes.

\footnotetext{
${ }^{2}$ Our model is also different in a number of other dimensions. For example, we do not impose a maximum life expectancy and we incorporate stochastic shocks.
} 


\section{Stylized facts: health and mortality over the lifetime}

\subsection{Mortality}

We study the evolution of mortality for a given cohort using data from the Human Mortality Database (hereafter HMD) . The HMD provides population and death counts by age, birth-year and gender collected through vital registration systems (birth and death certificates) and censuses, from 1816 up to 2015. Despite a few limitations, the HMD is the highest quality data available for cohort analysis. ${ }^{3}$ We compute mortality rates by age for each cohort as the number of deaths divided by the population at that age, and use these to compute survival rates. ${ }^{4}$ We also use these rates to compute cohort life expectancy. We focus on French cohorts for two reasons: these cohorts are large and the data goes back as early as 1816. While there are important differences across countries, as we discuss below the overall profiles of mortality by age are very similar for other countries.

It is important to distinguish cohort mortality from period mortality, which is used more often. Appendix Figure 14 summarizes the evolution of period and cohort life expectancy at birth by gender in France. Recall that period life expectancy is a synthetic construct computed using the cross-sectional mortality rates of all living cohorts in a given year. For example, to compute the 1850 period life expectancy, the mortality rates at age 70 are approximated using the observed mortality rates of 70 year-olds in 1850. In contrast, cohort life expectancy is computed using the realized mortality rates of a given cohort. To compute the 1850 cohort life expectancy, the mortality rates at age 70 are those observed in 1920. In a stationary environment, with stable mortality rates by age over time, the two measures are very close. For the 1816-1860 cohorts for whom life expectancy at birth was stable around 40 for females and 39 for males, the environment would appear to be close

\footnotetext{
${ }^{3}$ The HMD has some important limitations. Migration is not accounted for. Counts are not accurate for years during which the territory changed, in 1861, 1869, 1914, 1920, 1939, 1943, 1945, 1946 - often corresponding to wars (see Appendix 6). Data is imputed for ages above 90.

${ }^{4}$ Technically, we compute annual probabilities of dying at a given age instead of rates. Since the HMD provides no information on the distribution of births and deaths within a year, we make no adjustments for the fact that the deaths in the first year do not correspond to individuals born that year. The HMD reports probabilities $\left(q_{x}\right)$ that make adjustments based on a series of standard assumptions in epidemiology and demography. In order to avoid introducing discrepancies, we treat the data and the model symmetrically by computing death probabilities naively. These probabilities are very similar to the ones HMD computes (See Appendix Figure 13).
} 
to stationary. But life expectancy increased substantially starting in the late 19th century, with cohort life expectancy increasing more than period life expectancy, as would be expected when environmental or medical factors results in lower agespecific mortality rates. Females born around 1920 lived around 69 years, and males around 59, which is substantially longer than cohorts born a century earlier. Finally note that, although period life expectancy rose for men, several cohorts of men (born roughly 1880-1900) experienced declines in life expectancy, likely due to WWI and WWII.

The logarithm of mortality has the shape of a "tick mark": high at birth, low among the young, and high and rising almost linearly with age in late adulthood. This can be seen in Figure 1 which shows the logarithm of mortality rates by age, for selected birth cohorts of women born between 1860 and 1940 for various European countries (panel a) and for France (panel b). Although the level of mortality has changed subtantially over time, the basic evolution of mortality rates by age is very similar across many countries. These patterns are similar, though not identical for men (see Appendix Figure 15 and 16).

Mortality curves also display an "adolescent hump," especially visible in cohorts born in the 19th century. Starting in adolescence, mortality rates jump up. Hormonal changes and other changes associated with the transition into adulthood are thought to explain this adolescent hump (Preston et al. 2000, Thiele, 1871). Finally, there are clearly visible spikes for some cohorts, corresponding to wars and epidemics. These patterns are more visible when examining all the cohort curves (Appendix Figure 15 and 16). These patterns are not unique to humans. Bronikowski et al. 2011 show, using longitudinal data from primates living in the wild, that these patterns of mortality are very similar across all primates.

\subsection{Health}

A striking empirical pattern is that the distribution of health indicators is roughly gaussian, at any given age after birth and before old age. Partial but continuous health measures like birth weights and heights (which evolve until adulthood) are close to normally distributed too. For example, Wilcox and T Russell (1983) show that the distribution of birth weights is normal. That adults heights are normally distributed was shown in the 19th century first by Quetelet and then by Galton 
Figure 1: Mortality rates across populations
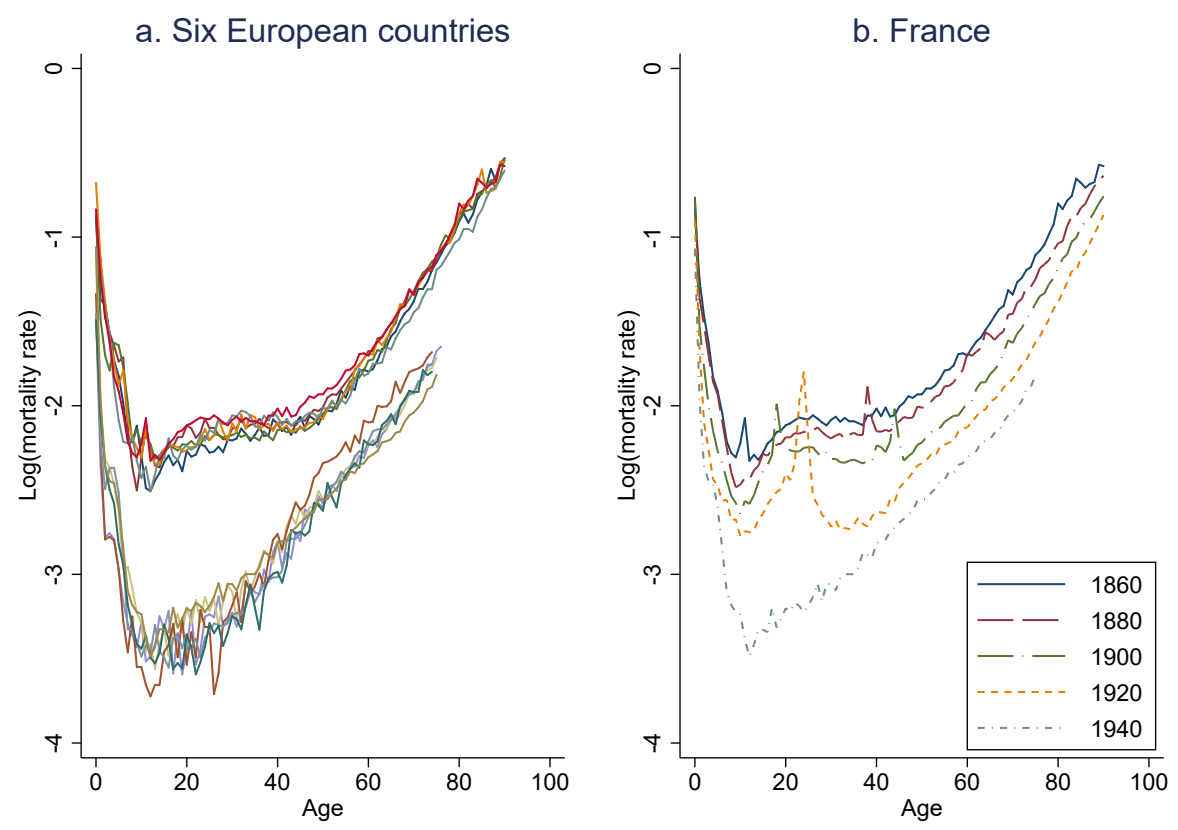

Note: Human Mortality Database. Panel a shows the $\log _{10}$ of the mortality rates by age for women born in 1860 and in 1940, in six European countries (Belgium, Denmark, the Netherlands, Sweden, France, and Norway). Panel $b$ shows the mortality rates for women born in France in 1860, 1880, 1900, 1920 and 1940.

and Pearson, as discussed by Tanner and Tanner (1981). ${ }^{5}$

How does the distribution of health evolve with age? Unfortunately, the HMD does not contain any health measure-there is in fact no data we are aware of that tracks a consistent measure of health from birth to death for a given cohort, but several studies provide partial descriptions of this evolution. Mean health falls with age, peaking sometime in young adulthood. For example Deaton and Paxson, 1998, Case and Deaton, 2005, Halliday et al. (2018) and Kaestner et al. (2020) show that self-reported health status declines with age among adults. Contemporary data show that hospitalization days (a proxy for morbidity or lack of health) are high in childhood, are at their lowest among individuals 12-18 and then rise again among older adults (see table P-10 in Centers for Disease Control and Prevention 2014). Deaton and Paxson (1998), Halliday (2011) and Halliday et al. (2019) also

\footnotetext{
${ }^{5}$ More recently Limpert et al. (2001) show that either a normal or a log normal distribution fits female heights well.
} 
report that the variance of health also rises with age, and then seems to level off or fall among the oldest, though the data are less clear about what happens among the oldest. The variance in organ function also rises with age (Steves et al. 2012). Lastly, both objective measures of health and subjective measures of health are strong predictors of mortality (Benyamini and Idler, 1999; McGee et al., 1999).

\section{A Unified Model of Aging and Mortality}

In this section we present a simple model that can account for these basic "stylized facts" about health and mortality. We will then show that our model fits mortality data well and investigate if it can generate other mortality patterns in the literature.

A basic model of natural mortality. Individuals are born with an initial health endowment $H_{0}$. This initial health endowment differs across individuals in the population and has an unknown distribution. Every period, the environment provides resources $I$ to all individuals, which increase $H$. In addition, individuals in the same environment are more or less lucky, and experience an idiosyncratic shock $\varepsilon_{a}$ to their resources. For example $I$ characterizes the per capita amount of food that a country produces, but a given person might receive less if for instance rain was unusually low in their location. The variance of $\varepsilon_{a}$ captures how unequal the distribution of resources within the population is. These idiosyncratic shocks are assumed to be i.i.d. every period. Finally, the health stock depreciates each period by an amount $d(a)$, which is increasing with age $a\left(d^{\prime}(a)>0\right)$ : every period there is a "user cost", reflecting, for example, errors in the epigenome, the system that translates genes into proteins, or damage to chromosomes occuring during cell division. Together these forces determine the evolution of the health stock, which is an unobserved latent variable.

Individuals die when their stock of health dips below a threshold $\underline{H}$, which is fixed throughout the lifetime and identical for all individuals. Let $D_{a}=\mathbb{I}\left(H_{a} \leq\right.$ $\left.\underline{H}, D_{a-1}=0\right)$ denote the random variable equal to one if the individual dies at age a. The population's health and mortality is characterized by the following dynamic system: 


$$
\left\{\begin{array}{l}
H_{a}=H_{a-1}-d(a)+I+\varepsilon_{a} \quad \text { if } D_{a-1}=0 \\
D_{a}=\mathbb{I}\left(H_{a}<\underline{H}, D_{a-1}=0\right) \\
D_{0}=0
\end{array}\right.
$$

with $I \in \mathbb{R}$. Note that if $D_{a}=1$ then $H_{a}$ is undefined - we do not observe the health of individuals after they die. But we observe the mortality rate for the population at age $a$, which is given by $M R_{a}=P\left(D_{a}=1 \mid D_{s}=0, \forall s<a\right)$. Thus the distribution of health at any age is a function of the entire history of shocks and investments, as can be seen from the definition of $M R_{a}$, which conditions on survival in every previous period.

We make three key parametric assumptions, in order to make the model more tractable and consistent with the empirical evidence about the evolution of health with age. First, $H_{0}$ follows a normal distribution $\mathcal{N}\left(\mu_{H}, \sigma_{H}^{2}\right)$. Second, shocks to resources every period also follow a normal distribution $\varepsilon_{a} \sim \mathcal{N}\left(0, \sigma_{\varepsilon}^{2}\right) .{ }^{6}$ Third, depreciation is a power function $d(t)=\delta a^{\alpha}$ with $\delta \in(0, \infty), \alpha \in(0, \infty){ }^{7}$ This aging process in the model starts directly at birth, consistent with evidence that aging markers are evolving among children (Wong et al., 2010), and it increases with age, as in biological models of senescence (Armitage and Doll, 1954; Pompei and Wilson, 2002). ${ }^{8}$ Figure 2 illustrates the evolution of health and mortality in the first two periods. Initially, the health distribution is normal. Then the health distribution shifts to the right during the first period (as long as $I$ is positive and larger than the aging term) and spreads out (because of the stochastic shock $\varepsilon_{a}$ ). Individuals who were born too frail or who experience large negative shocks move to the left of the threshold and die. Graphically, the infant mortality rate (the fraction of individuals who die in the first period) corresponds to the area under the dashed red curve below the threshold. In the second period, this truncated distribution moves right again (if $I$ is large relative to $d(1)$ ), and the population receives a new shock, generating mortality again among those with large negative shocks. ${ }^{9}$

\footnotetext{
${ }^{6}$ Conceptually, the model has no difficulty accommodating other distributions. But simulations with alternative assumptions (e.g. log normal errors) resulted in counterfactual mortality rates and a poorer overall fit.

${ }^{7}$ Our estimates for human populations find that $\alpha>1$ and the depreciation is therefore convex in age, as hypothesized by Grossman. This is not imposed a priori by the model.

${ }^{8}$ See Gavrilov and Gavrilova (1991) and Weibull (1951) for attempts at biological microfoundations drawing on reliability theory from engineering.

${ }^{9}$ If, in the first period, depreciation were very large relative to investment, then mortality would
} 
Figure 2: Health and mortality in the first two years of life
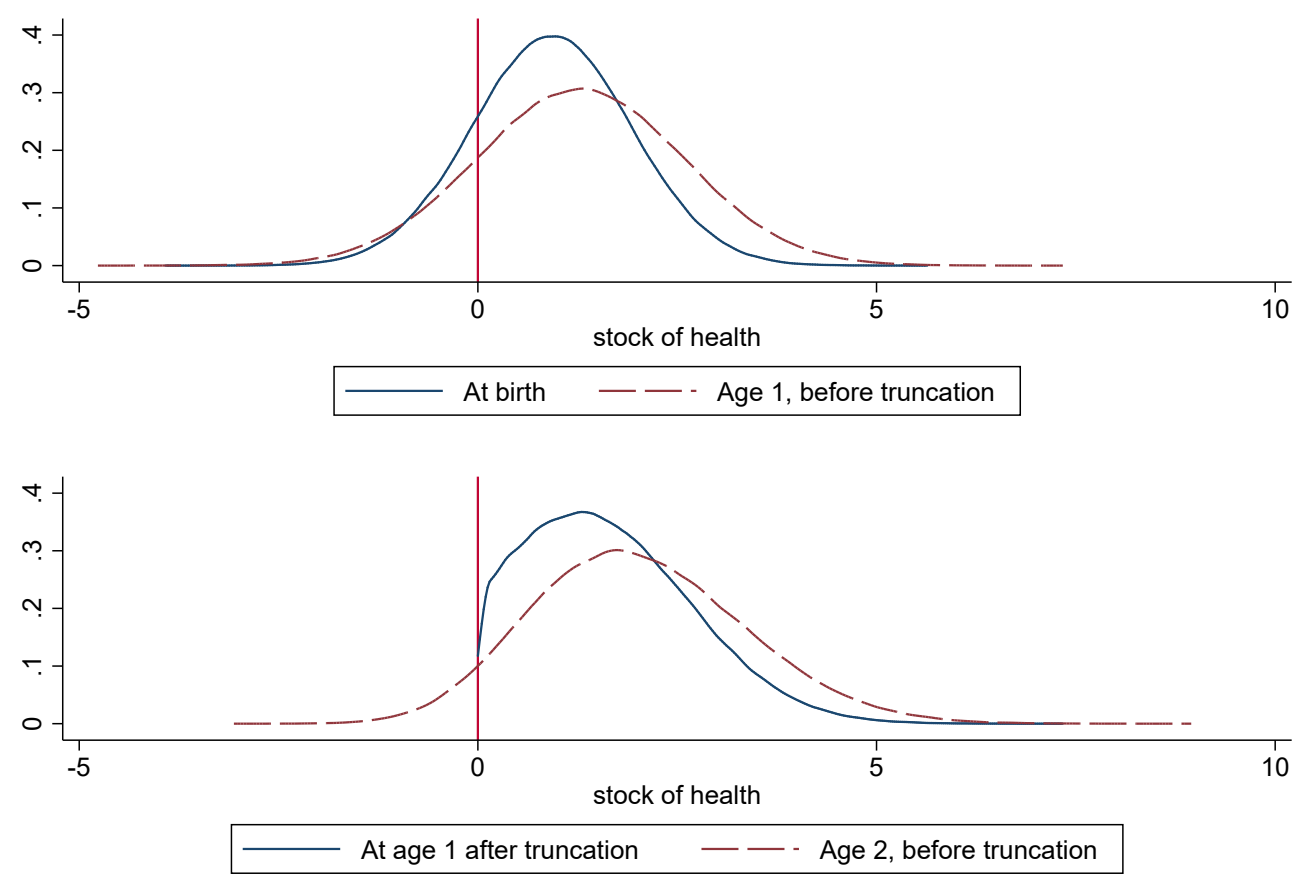

Data from simulations

The stochastic term $\varepsilon_{t}$ therefore plays a key role. In its absence, there would be no deaths in period 2 - nor in any subsequent period, until the depreciation term becomes large enough to push the leftmost part of the distribution below the threshold. ${ }^{10}$ Then mortality would increase every period. Eventually everyone dies-this is proved more formally in Appendix $6 .{ }^{11}$

rise from birth onwards - a theoretical possibility observed neither among humans nor primates.

${ }^{10}$ If $I$ is less than aging, then one could also generate positive mortality in the second period without a stochastic term. But then mortality would be rising from age 2 onwards, which we do not observe in the data.

${ }^{11}$ This feature is different from the standard Grossman model in which eternal life is possible, as noted by Case and Deaton (2005) or Strulik (2015). 
Figure 3: Model behavior

(a) The evolution of the health distribution over the lifetime

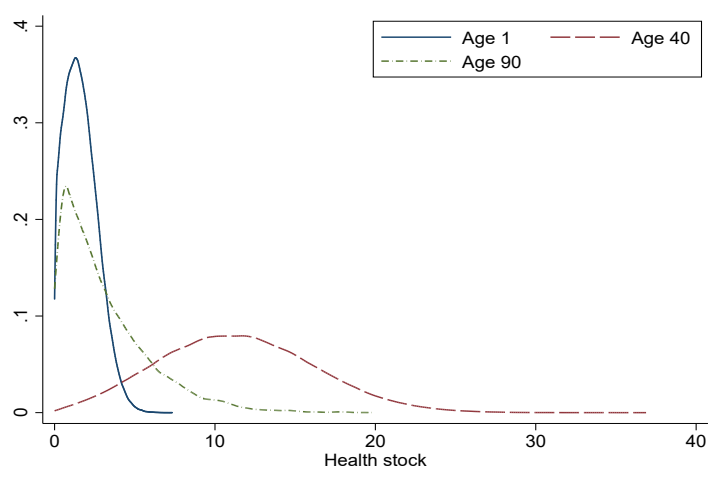

(b) Age profile of population health and mortality

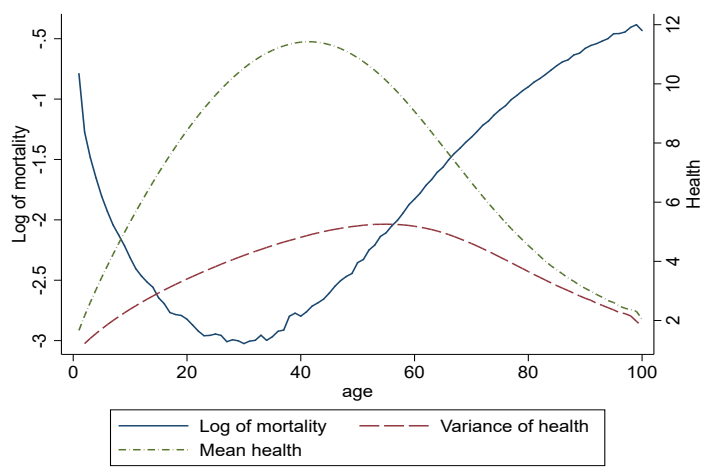

Note: Simulated data for a population of 500,000 individuals. For this simulation we use the following parameters: $I=0.3575753, \delta=0.0004789, \sigma=0.8353752, \alpha=1.7883, \mu_{0}=0.925079$. Panel $a$ shows the density of health for the population at ages 1, 40 and 90. Panel $b$ plots the average health, the variance of health and the mortality rates of the population over the lifetime.

This basic model matches the stylized patterns described above well. Figure $3 \mathrm{~b}$ shows the evolution of the health distribution and the resulting mortality over the lifetime. Just like their empirical counterparts, cohorts in our model exhibit the following characteristic patterns: (1) the distribution of health is roughly normal in most periods; (2) mean population health increases and then falls (conversely the model generates morbidity rates that are a U-shaped function of age $)^{12}(3)$ the variance of health increases and then falls; and (5) mortality falls and then rises at a roughly log-linear rate after middle age. There is only one feature of the data that

\footnotetext{
${ }^{12}$ Morbidity rate is the fraction of individuals with health below a health threshold but above the death threshold.
} 
we have not accounted for: the increase in mortality around adolescence ("adolescent hump") which we consider next.

External causes of death. Not all deaths have direct biological causes. Many deaths, like accidents or homicides, strike individuals regardless of their health status. These "extrinsic" causes of death can be integrated in the model by simply adding an i.i.d. "accident shock" that is independent of the stock of health $H_{a} \cdot{ }^{13}$ Then a constant fraction $\kappa \in[0,1]$ of the population is randomly killed every period. This random accident rate places a floor in the level of mortality that is constant across ages. ${ }^{14}$ Figure 4 a shows what happens to health and mortality when we add a lifetime accident shock. This extended model also replicates the basic shape of mortality well-a constant accident rate increases the level of mortality but does not change its basic evolution, nor does it affect the distribution of health among the living.

\footnotetext{
${ }^{13}$ Corporate default models similarly complement the equation describing the evolution of firms' values with a "jump to default" component.

${ }^{14}$ If all health-related deaths were eliminated, this accident rate would uniquely determine the life expectancy of the population $(1 / \kappa)$.
} 
Figure 4: Adding accidents to the baseline model

(a) Adding accidents to the model

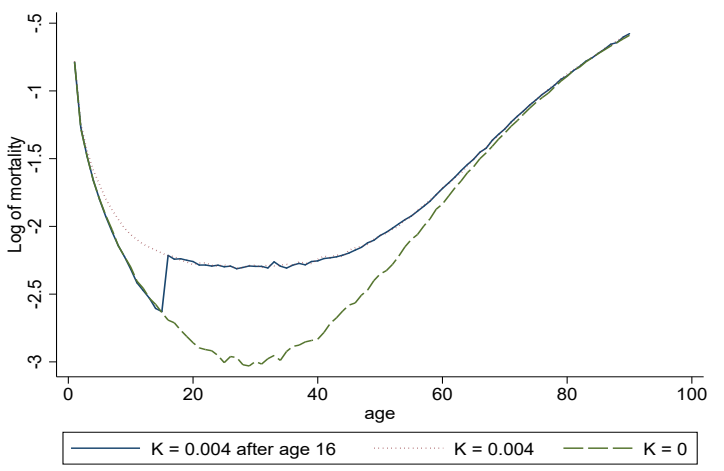

(b) US Mortality rates per 1,000 in 1990, by cause of death

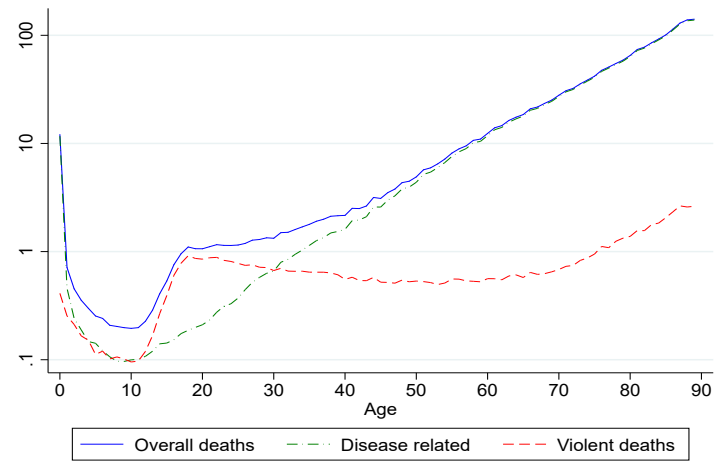

Note: Figure a: The baseline parameters are the same as in Figure 3b. The red dashed line shows the mortality curve of a population that experiences a 0.005 percent chance of dying every period due to an accident, unrelated to health. The dotted green line shows the model which assumes the accident rate is zero at birth but jumps to 0.005 in adolescence. Mortality rates are higher as a result of external deaths but more so in middle ages because of competing risks: older individuals that are hit by an accident shock are also unhealthy and would die even in the absence of an accident shock. Figure b is reproduced from Schwandt and Von Wachter (2019) who generously agreed to let us use it. The data come from period (not cohort tables) so they are not directly comparable to ours. But we use it to demonstrate that the mortality rate from non-disease related causes of death is well approximated by a step function that turns on in adolescence. Mortality rates are shown in $\log (10)$ scale.

Contemporary data however show that the mortality rate from external causes of death is not constant throughout life. Instead, it is well approximated by a step function, with a major increase around adolescence (Figure $4 \mathrm{~b}$ ). ${ }^{15}$ Based on this evidence, we assume that $\kappa$ starts at zero but becomes positive in adolescence at an arbitrary age $\left(a^{*}\right)$. Adding external causes of death in this fashion adds two more parameters to the model. For simplicity, we assume that the onset of adolescence is unaffected by health levels, and we take it to be exogenous throughout the paper we use historical data on the onset of menarche to identify the onset of adolescence instead. ${ }^{16}$ Figure 4 a shows that adding this step function results in a profile of

\footnotetext{
${ }^{15}$ The shapes in the two figures are not identical. However, the contemporary data is not cohort but period data. In contemporary settings, the two profiles differ substantially, as discussed already. However there is no historical cohort mortality data by cause of death, so we cannot create the more relevant cohort figure.

${ }^{16}$ This assumption could be relaxed. The onset of menarche, a proxy for adolescence in women, has declines from approximately age 16 to age $12 \sin$ the last two centuries. This development has been linked to nutritional changes and might be a function of health.
} 
mortality that qualitatively matches the main features we observe. ${ }^{17}$

\section{Explaining Mortality Patterns}

We now assess whether the model can quantitatively match observed patterns of mortality. We do this by estimating the parameters of the model and assessing the model's fit for both human and primate cohorts.

\subsection{Identification and estimation.}

Identification. Two out of the nine parameters of the full model cannot be identified. To see this, note that the expression for mortality in the first period $M R_{1}=$ $P\left(D_{1}=1\right)=P\left(\mu_{H}-\delta a^{1}+I+\varepsilon_{1}<\underline{H}\right)$ is the standard Probit model. We can subtract $\underline{H}$ and divide by $\sigma_{H}$ on both sides of the expression that determines the probability of dying, and leave the mortality rate unchanged. Therefore the threshold $\underline{H}$ and the standard deviation of the initial distribution $\sigma_{H}$ are not identified. Without loss of generality, we set $\underline{H}=0$ and $\sigma_{H}=1 .{ }^{18}$ After normalization, all the parameters are expressed in "standard deviation" units, except for $\alpha$ and $\kappa$, which are "scale free" - they do not depend on the initial distribution. For example, we interpret $\mu_{H}$ as the distance from the threshold of the initial distribution, in standard deviations of the initial distribution.

The rescaled model characterizes the biological evolution of health and mortality of a cohort using 7 (rescaled) parameters: one for the mean initial health $\left(\mu_{H}\right)$, two governing the aging process $(\delta, \alpha)$, two characterizing the effects of resources, in the form of average investments $(I)$ and the variance of these investments or shocks $\left(\sigma_{\varepsilon}^{2}\right)$, and finally one $(\kappa)$ capturing the accident rate increase occurring in adolescence occurring at $a *$. We do not estimate this last parameter. For humans, we assume adolescence starts at age $=(-0.0175 \times$ calendar year $)+47.4$ for all women, based on the estimates provided in de La Rochebrochard (2000) who

\footnotetext{
${ }^{17}$ Note that, regardless of whether the accident rate is constant or increases in adolescence, the distribution of health and its evolution over the lifetime remain unchanged and follow the patterns shown in panel $b$ of Figure $3 \mathrm{~b}$. This is because these deaths are random and do not depend on health status.

${ }^{18}$ More precisely, we need to normalize 2 out of three parameters. We find it more intuitive to normalize the threshold rather than the initial mean, but this choice is arbitrary.
} 
estimated the equation using historical data from multiple sources. Adolescence is assumed to start one year later for men, as observed in contemporary settings. We test the robusteness of the results to alternative assumptions. For chimpanzees we use two alternative start dates, age 8 and age 14, which span the ranges described in the literature. ${ }^{19}$

Estimation. Despite the model's conceptual simplicity, the mortality rate at a given age cannot be expressed in closed-form..$^{20}$ We therefore estimate the parameters using the simulated method of moments. We matched the annual age-specific survival rates, and thus implicitly, life expectancy. We construct survival curves using the population and mortality counts by gender, year of birth and year of death for France. Appendix A has the data and estimation details.

\subsection{Mortality rates over the lifetime}

We start by estimating the model for the 1816 cohort. The model very closely matches the 1816 cohort's mortality rates at every age (Figure 5a shows results for females). For females, the predicted life expectancy is 38 years and 102 days compared to the actual life expectancy of 38 years and 91 days. ${ }^{21}$ We estimate an initial mean health of about 0.86 , so many individuals are born at or below the threshold (Appendix Table 1). Absent any shocks or investment in the first period, infant mortality would have been roughly $15 \%$ (instead of $17 \%$ ). Mortality falls dramatically after age 1 because there is selection (many frail individuals have already died), and because investment is large relative to aging in the first period ( $I$ is estimated as 0.4 and $\delta$ as 0.0006 ). The variance of resources is large (estimated to be roughly 1) so a few unlucky individuals still fall below the death threshold after age 2. We estimate an external mortality rate of roughly 9 per thousand for every year after adolescence starts, lowering the 1816 cohort's life expectancy by about 7.6 years. ${ }^{22}$ This provides an upper bound estimate of the effect of maternal mor-

\footnotetext{
${ }^{19}$ Bronikowski et al. 2011 report the onset at age 14, other sources (Behringer et al. (2014)) place the onset at age 8 .

${ }^{20}$ Our discrete model is similar to a class of models used for corporate default probability and securities pricing. This literature has established that, except for the particular case of a constant or linear drift, these models do not admit closed-form solutions (see Lando, 2004).

${ }^{21}$ Appendix Table 1 shows alternative measures of fit and the estimated parameters.

${ }^{22}$ The fit of the model is poor around the time of adolescence. This can be improved upon by allowing the onset of adolescence to be a normally distributed function and estimating its parameters. These results are shown in Appendix Table 3.
} 
tality - the main cause of death for women in the 19th century - on life expectancy in the past. Accounting for external deaths is improtant - the fits of the model improves very substantially when we do. The estimated parameters also change substantially.

Log mortality starts a steady increase after age 45 . This occurs because while $\delta$ is small $(\sim 0.0006)$ the aging rate $\alpha$ is around 1.8 , so that the aging function $\delta t^{\alpha}$ is increasing more than linearly with age. Because health resources $I$ are increasing only linearly, eventually all individuals die, even lucky ones with many large positive health shocks. ${ }^{23}$ These results are robust to a number of alternative estimation modifications including using alternative weights, using an alterantive objective function, and allowing for truncation at age 90 . We also estimates models where we estimate the onset of adolescence is normally distributed, or where we estimate the distribution of the onset of adolescence. These results are shown in Appendix Table 3 and they show that the fit is not very sensitive to these alternatives.

Gender differences. Appendix Figures 17a show the results for males born in 1816 and Appendix Table 1 shows the estimated parameters. Men born in 1816 lived shorter lives than women, as has been documented before. Consistent with their greater frailty and higher infant mortality rates, males' inital mean health is 19\% lower than that of females' (Goldin and Lleras-Muney 2019, Cullen et al. 2016). There is a substantial increase in deaths in adolescence for both males and females, but it is larger for men, consistent with their greater involvement in accidents and violent deaths. However, because males have higher overall mortality rates, the elimination of accidental deaths would increase their life expectancy by about 7.6 years, very similar to the predicted gains for women.

Accounting for the adolescent hump significantly affects the estimated parameters and drastically improves the fit, as was the case for women. After accounting for the adolescent hump (column 2 of Table 1), we find that males receive slightly larger annual investments (about $10 \%$ greater) but also experience greater variance in investments. They also age faster in old age (though women age a bit faster during prime ages). Overall, the model fit is excellent for both genders, though the fit is slighter better for females.

Primates. Human mortality patterns are very similar to those of other primates.

\footnotetext{
${ }^{23}$ See Appendix 6 for a rigorous proof. This statement holds even in the absence of external deaths.
} 
Figure 5: Model fit for humans and primates

(a) French women born in 1816

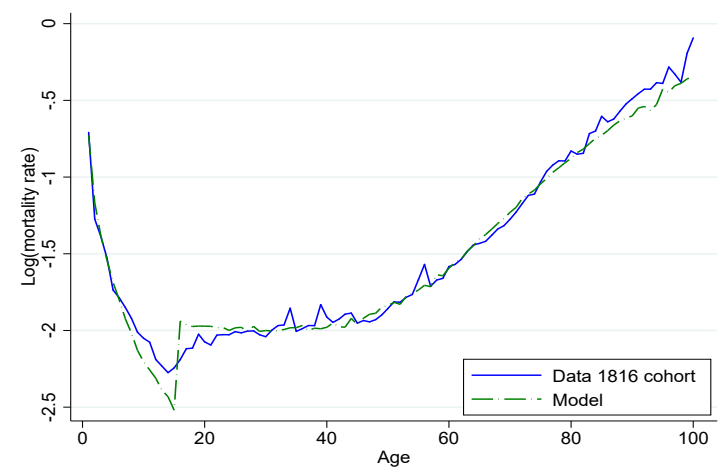

(b) Female chimpanzees

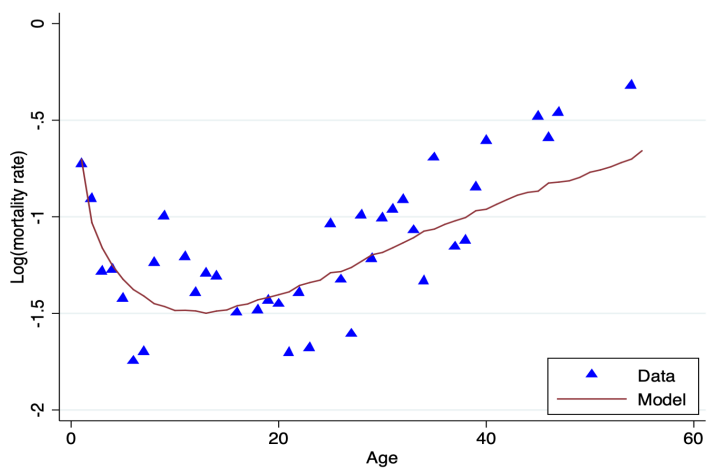

Note: Panel a shows the data and estimated curve for French women born in 1816. Panel $b$ shows the data and estimated parameters for female chimps. Appendix Tables 1 and 2 show the estimated parameters.

Therefore our basic model should be able to describe primate mortality well, particularly since they live in relatively stable environments, experience no technological change and have few optimization opportunities. We estimate the model using the best available data on populations of female chimpanzees living in the wild from Bronikowski et al. (2011). These populations are tracked in the wild from birth to death and have been used to compare mortality rates across various primate populations. We focus on chimpanzees because they are the closest primates to humans.

Figure $1 \mathrm{~b}$ shows the results for females chimps. We still obtain a very good fit, despite the small populations and therefore much noisier estimates. The parameter estimates are provided in Appendix Table 2. Compared to human females, female chimps are born in better health, consistent with the observation that human infants are born frail relative to other species. ${ }^{24}$ We also estimate a much lower rate of accidental deaths among female chimps starting in adolescence, in line with the fact that maternal mortality is a uniquely important problem among humans (Rosenberg, 1992). ${ }^{25}$ But other parameters favor longevity among humans. In fe-

\footnotetext{
${ }^{24}$ There are several theories for this-for a discussion, see Rosenberg and Trevathan (1995).

${ }^{25}$ As Rosenberg (1992) puts it, "most primates experience parturition as a simpler, shorter, and very likely less painful process (than humans)." This difficulty is believed to have led to the use of birth attendants in almost all known human cultures and times. Our estimates do not imply that external causes of death are unimportant among primates-neither model estimates a baseline
} 
male chimps, the estimated annual investment is about $20 \%$ smaller and the variance of this investment is 10\% larger than among human females. Most notably, $\delta$ is much larger ( 0.06 v. 0.0006) than in humans, resulting in much faster aging. As in humans, female chimps live longer than males, partly because males have larger external causes of death than females. ${ }^{26}$ They also have larger annual investments, larger variance in resources and larger aging $(\alpha)$ than females. But unlike humans, males have larger initial health.

\subsection{The rectangularization of survival and the sources of increases in life expectancy}

Remarkably, the model is able to track the evolution of the mortality profiles for all the individual cohorts since 1816. This evolution is characterized by a "rectangularization" of the survival curves, which has accelerated over the last decades. Panel a in Figure 6 shows the rectangularization of the survival curves of French women born between 1816 and 1947. Survival to age 1 has increased dramatically. The next section of the survival curve - roughly from age 1 to age 60 - has considerably flattened. In addition, a steep downward slope has emerged among the oldest. As a result, more than $70 \%$ of those born in 1940 live past age 70, whereas in the 1816 cohort fewer than $30 \%$ did. Panel a in Figure 6 shows that the model captures this rectangularization with great accuracy: the observed (blue markers) and estimated (red dashes) survival curves are very similar. The model can fit the data for the 1940 cohort almost as well as for the 1816 cohort. The results are similar for men (Appendix Table 4) but we do not discuss them here for brevity. ${ }^{27}$

What are the sources of increases in longevity according to our estimates? Panel $b$ in Figure 6 shows the evolution of four of the estimated parameters from 1816 and 1923. Starting in the 1830s, we see a constant and rather drastic decline in external causes of death, which is consistent with the elimination of maternal mortality (a major cause cause of death among prime-age women in the past (Loudon, 1988)), and with the steep decline in violent deaths as documented for instance by

accident rate throughout.

${ }^{26}$ Not surprisingly, correctly timing the onset of adolescence is not important for females, but makes a substantial difference for males.

${ }^{27} \mathrm{~A}$ full examination of gender differences in the estimated time series is beyond the scope of this paper. 
Pinker (2011). Health at birth, $\mu_{H}$, started to increase steadily only at the end of the century, consistent with the timing of improvements in water, sanitation, and the elimination of epidemic and infectious disease mortality, which greatly reduced infant mortality (Cutler et al., 2006; Preston and Van de Walle, 1978).

By contrast, health resources $(I)$ did not change much in the 19th century (they fall a bit and rise again), consistent with the debate on the questionable benefits of the Industrial Revolution on health and living standards. However, there is a steady decline in the variance of health resources - it is also unclear why this occurred, though it is possible food availability became less variable. Alternatively it might be difficult for the model to separately identify the effects of $I$ from the effects of its variance because the data on mortality is only informative about the left tail of the health distribution. Most interestingly, we observe a substantial decrease in the force of aging (see Figure 6c), the causes of which are unclear. Since food consumption and heights were rising, this suggests that nutrition is a possible determinant of the aging function (Fogel, 1994). ${ }^{28}$

Appendix Figure 18 shows the performance of the model for each birth cohort born 1816-1923 (the last cohort with complete data up to age 90). The fit is in general excellent and is steady throughout the 19th century, but it gets much worse for cohorts born after 1900. There are a few reasons for this. First, for some of the more recent cohorts, there is still some potential bias due to censoring. Second, three events in the early 20th century are likely to severely affect the cohort profiles: WWI, the 1918-1919 Spanish flu pandemic, and WWII. We discuss below how we estimated these, but these events are difficult to model. The data during these episodes is also of significantly lower quality, as changes in territory, for example, make the computations of death rates difficult. Lastly, we are assuming that there is no inter-temporal optimization of health investments taking place. The rise of social insurance programs throughout the 20th century suggests that this simplifying assumption is likely to be violated for more recent cohorts. We discuss optimization and its effects in the last part of the paper.

\footnotetext{
${ }^{28}$ There is unfortunately very little data to investigate what factors might affect the estimated parameters. This is an interesting area for future research.
} 
Figure 6: Evolution of survival for French females born 1816-1940

(a) Observed and Estimated Cohort Survival Rates

(b) Parameter evolution
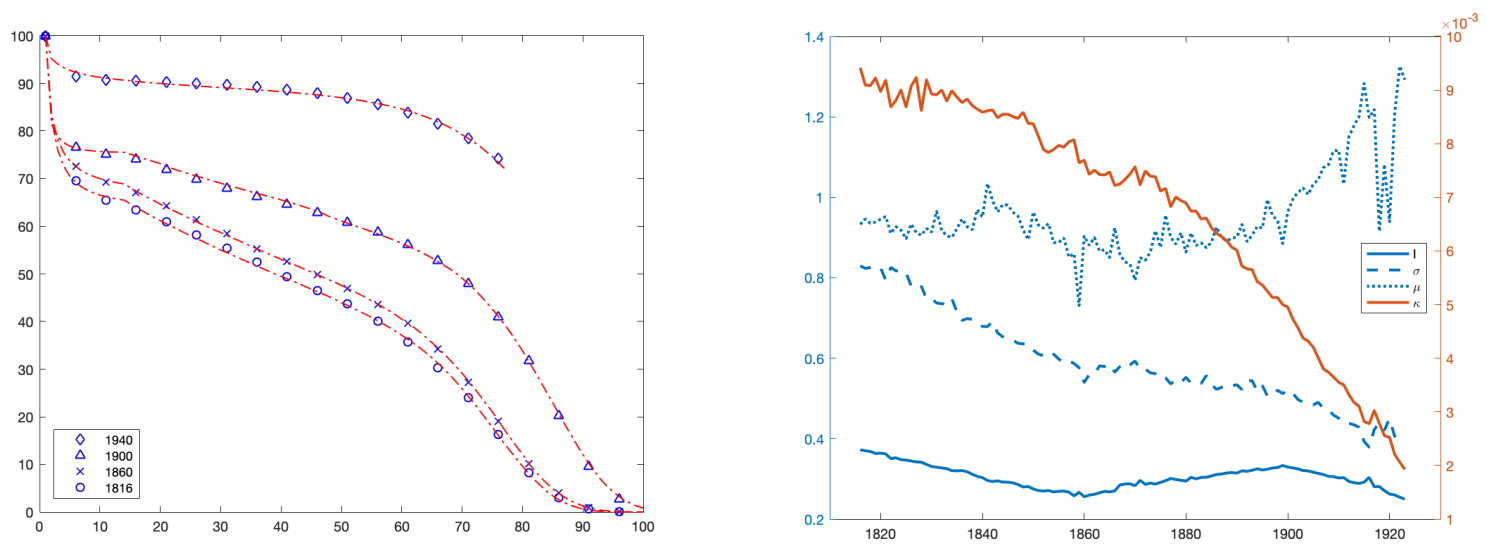

(c) Estimated Aging Function for the 1816 and 1916 cohorts

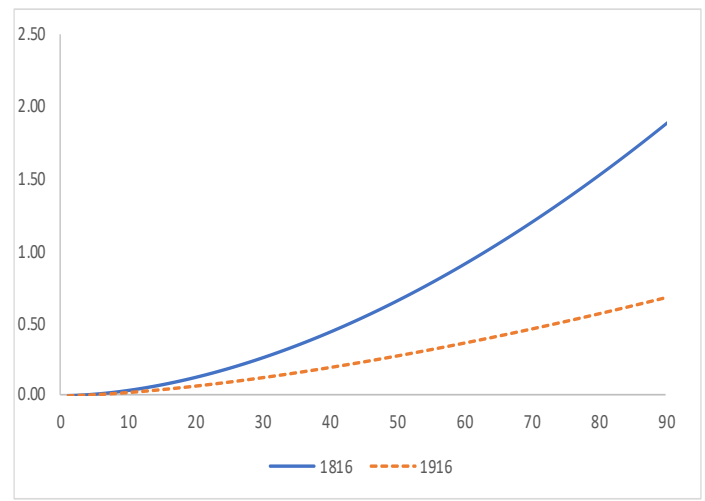

Note: Panel a shows the observed (blue markers) and estimated (red dashes) survival curves for four cohorts of French women born about 40 years apart between 1816 to 1923 . The y-axis shows the survival rate and the $x$-axis shows the age. Panel $b$ displays the evolution of estimated parameters, except for the aging parameters which are shown in Appendix Figure 6c. On the left axis are the values for the three blue lines corresponding to resources $(I)$, the variance of the lifelong shock $(\sigma)$, and initial health at birth $(\mu)$. The accident rate $\kappa$ in red is on the right axis. The model is estimated separately on each cohort. We treat the two World Wars as two independent negative resources $I$ shocks as discussed in the next section, see Appendix $C$ for details. Panel $c$ plots the estimated aging function $\delta * t^{\alpha}$ for the 1816 cohort $\left(0.0006 \cdot t^{1.79}\right)$ and the $1916\left(0.0007 \cdot t^{1.53}\right)$ cohort. It shows that the aging rate has flattened dramatically due to a $15 \%$ decline in $\alpha$. 


\section{Understanding Mortality Dynamics}

The evolution of the parameter estimates for each cohort suggests large, lasting changes in the environment but does not identify their sources. In this section, we investigate how environmental changes and their implications on mortality rates can be understood, through the lens of the model, as proceeding from simple shocks to the model parameters. Although there is no data to estimate a more sophisticated model that considers how environmental factors affect the model's parameters, we conduct a series of qualitative exercises to demonstrate that the model can rationalize the effects of temporary and permanent shocks in the environment.

\subsection{Socio-Economic Status Mortality Gradient}

A substantial literature documents health and mortality "gradients" - large and persistent differences across individuals with different levels of socio-economic status such as education, income level, occupation or race (Cutler et al., 2012). For instance, Americans with a high permanent income level at age 40 have lower subsequent mortality relative to those with lower incomes (Chetty et al. 2016). Figure 7a reproduces Chetty et al. (2016)'s figure showing that the log mortality curves partly converge in old age (implying smaller gaps at older ages in percentage terms). Similarly, more educated individuals tend to have healthier behaviors (Cutler and Lleras-Muney 2010), resulting in lower mortality rates throughout. In their review, Hummer and Lariscy (2011) write, "analyses invariably show that educational disparities in mortality are narrower at older than at younger adult ages."

How can the model rationalize such gradients? Suppose that we extend our model so that lower income leads to lower $I$ throughout life. In other words, assume there exists a function $I=I(Y, E)$ with $I^{\prime}>0$ for all inputs such as income $Y$ or education $E$. What is the effect of increasing $Y$ thoughout the lifetime on mortality rates?

We illustrate this by simulating the effect of lowering $I$ by $50 \%$ on the 1816 French female cohort. Figure $7 \mathrm{~b}$ shows this results in higher and flatter log-mortality curves for the poorer population. Moreover, the curves for the rich and the poor converge in old age, just as documented by Chetty et al. (2016) and shown in Figure 
$7 \mathrm{a}$, and consistent with the evidence of education. This occurs because, although the frailest individuals are saved in the first period when $Y$ increases, $Y$ shifts the distribution of health right for all individuals in the second and subsequent periods. Therefore overall mortality rates fall throughout the lifetime.

When looking at the profile over the lifetime, the narrowing of the mortality SES gradient (in percentage terms) occurs in the model only after a certain age. ${ }^{29}$ To illustrate this, Figure 7c shows the effect of greater $I$ on the gap in mortality between the rich and the poor, expressed either in levels or logs. Each point in the figure plots the difference between the rich and the poor at a given age - this is equivalent to plotting the age-specific coefficients from a regression of age-specific mortality (or log mortality) on a dummy for the rich population. In log terms, this mortality gap initially grows with age, as hypothesized by the cumulative advantage hypothesis (Lynch 2003, Ross and Wu 1995), because greater $I$ pushes the entire population further and further away from the threshold every period. However, gradients eventually fall because of selection, as suggested by Crimmins (2005): the population with lower $I$ starts dying, leaving only the healthiest individuals alive. The figure also shows that in levels, SES gaps in mortality rates are $\mathrm{u}$-shaped, instead of hump-shaped, with age. The reason the gap diminishes between childhood and adolescence is that, when $I$ is high relative to aging, fewer and fewer people are close to the death threshold. Thus SES gaps are very small among prime adults, and possibly hard to detect in finite samples, but they rise with age as illustrated by Kaestner et al. (2020) for education.

Health. Lower income (or education) and thus lower $I$ is also predicted to lower average health at all ages. But the effect increases with age, and then declines once mortality starts rising in both levels and percentage terms. (See Figure 7c.) These predictions match the evidence in Case et al. (2002), Currie and Stabile (2003) and House et al. (2005), who show that the gaps in self-reported health status and morbidity between those born in poor families and those born rich families grow with age, but decline after 65 .

Resource scarcity or accelerated aging? Instead of affecting annual resources, higher SES could instead lower rates of aging. SES is associated with more frequent physical exercise, lower exposure to pollution or lower stress which could

\footnotetext{
${ }^{29}$ Note that the gap in levels between the high and low $I$ populations is hump shaped with age instead of U-shaped, growing with age among older adults (Figure 7c).
} 


\section{Figure 7: SES gradients}

(a) Persistent SES Mortality gap in Chetty et al.

(2016)

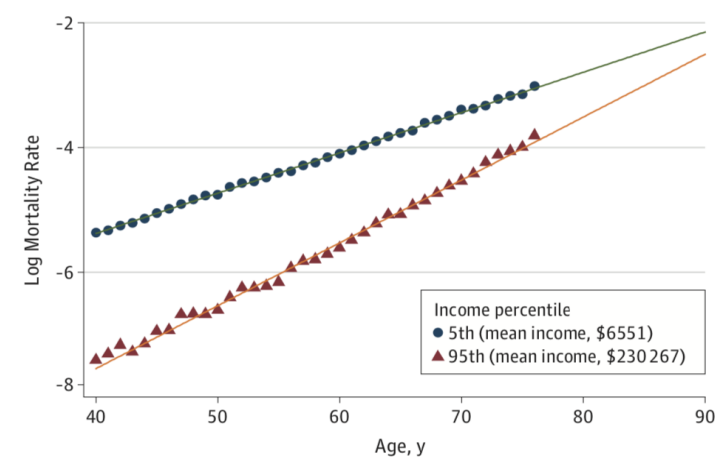

(c) Effects of decreasing $I$ by $50 \%$ on mortality

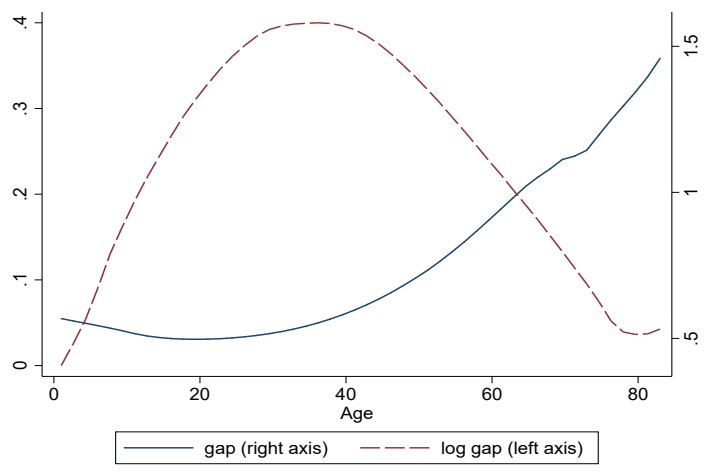

(b) Persistent SES Mortality gap in our model

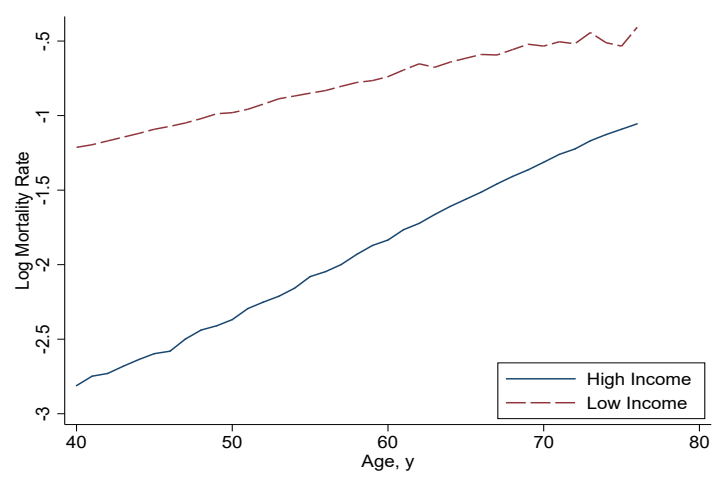

(d) Effects of decreasing $I$ by $50 \%$ on health

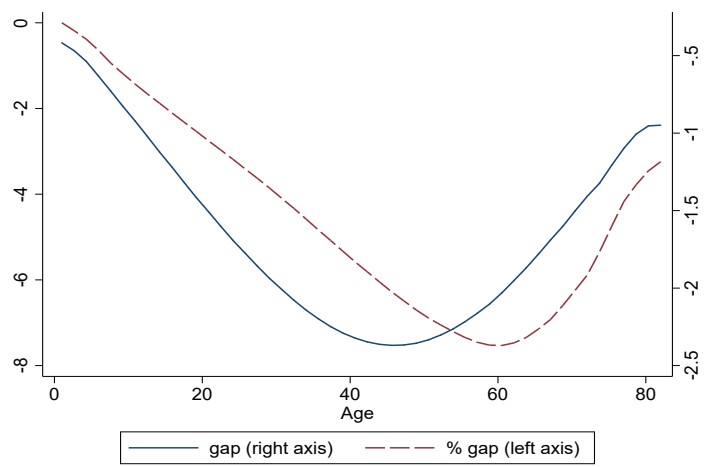

Note: Panel a reproduces the results from Chetty et al. (2016). Panel $b$ shows the predicted mortality rate for the 1816 cohort (using the parameters from in Appendix Table 1 but setting the accident rate at 0 throughout for simplicity) and the counterfactual mortality that results from a 95\% decline in $I$ for this population. The baseline 1816 cohort is labeled "High Income" and the counterfactual population is labeled "Low Income." Panel c shows the simulated effects of increasing the baseline level of $I$ by $50 \%$ on mortality in both levels and percentage terms. We plot the gap between the baseline and the affected population. This gap is computed as MR(low)-MR(high), or $\mathrm{H}(\mathrm{low})-\mathrm{H}($ high). Panel $d$ shows the effects of increasing the baseline level of $I$ by $50 \%$ on mortality on health. The baseline parameters are the same as in Figure 3b. 
be conceptualized as affecting the rate of depreciation. In the model, an increase in the aging parameters ( $\delta$ or $\alpha$ ) and a decrease in $I$ generate similar health and mortality profiles among the old, as shown in Appendix Figure 19. Thus, with data from (mature) adults only, it is not possible to infer whether SES is affecting annual resources $I$ or aging rates. But higher aging rates do not result in any visible health or mortality gaps among children, whereas higher $I$ does. Therefore, the evidence in Case et al. (2002) or Currie and Stabile (2003), interpreted through the lens of the model, suggests that changing family income is equivalent to changing $I$. It is possible to break this observational equivalence by relating measures of aging to SES. Liu et al. 2019 find that education and race are associated with lower methylation rates (a biomarker for aging), suggesting SES also affects aging rates.

Before moving on, we note that it would be ideal to estimate our model using cohort data by education or income - however, there is no data that we know of that allows one to track cohorts from birth (or age 25) to death by permanent income or education levels. ${ }^{30}$ Our simulations only show that the model can rationalize the observed patterns in the data.

\subsection{Non-Monotonic Effects of In-Utero Shocks}

Detrimental events in-utero (famines, war, stress, etc.) result in large and persistent declines in health that are visible in infancy and old age (Almond and Currie, 2011) and in elevated mortality among the survivors. ${ }^{31}$ Suppose again that we allow for the initial mean of the distribution, $\mu_{H}$, to be affected by outside forces. What is the effect of exogenously lowering initial health on the subsequent health and mortality of the survivors? We use the 1816 parameters as a baseline to simulate this effect.

Our model rationalizes why the age-profile of these responses is non-monotonic, which canonical models have been unable to explain. The empirical literature finds that the effects of various shocks appear to "fade out" initially, only to re-appear

\footnotetext{
${ }^{30}$ There are a few longitudinal data sets tracking individuals from birth onwards, but they do not provide annual data.

${ }^{31}$ Van den Berg et al. $(2006,2009)$ and Masters $(2018)$ show that being born in a recession is associated with increases in mortality rate later in life. Similarly, Lindeboom et al. (2010) show that children born during the Dutch Potato famine lived shorter lives as a result of the famine. Bharadwaj et al. (2013) show that investments made immediately after birth among low birth weight children result in lower mortality rates later in life.
} 
Figure 8: The effects of negative in-utero shocks

(a) Monotonic response in Grossman's model

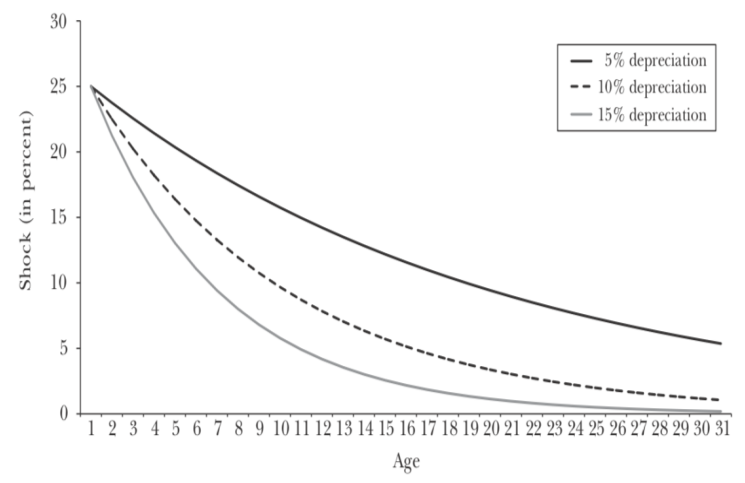

(b) U-shaped decreases in health in our model

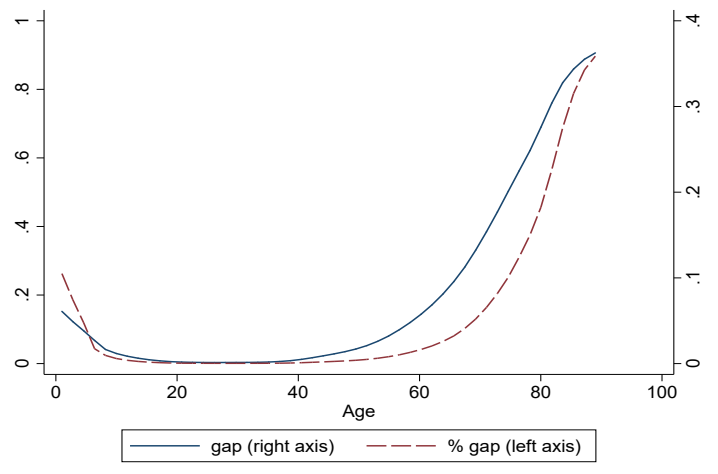

(c) Negative in-utero shocks increase lifetime mortality

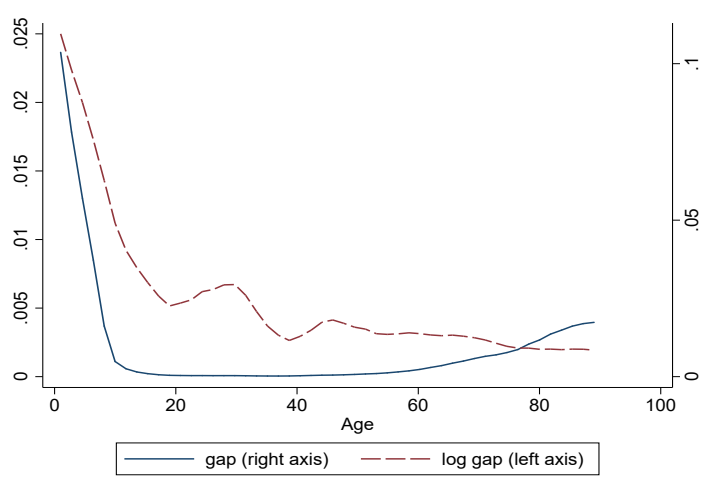

Note: Panel $a$ is reproduced from Almond and Currie (2011) and shows the decline in the health stock due to a shock in utero that is predicted by the standard Grossman model. This effect is initially large but it fades over time and will be close to zero among adults older than 30. Panel $b$ shows the simulated effects of a 50\% decline in in-utero health for the 1816 French population in the model (setting the accident rate at 0 throughout for simplicity). The figure plots the decreases in health, in either levels or percentage terms. Panel $c$ shows the effects on mortality in both levels and percentage terms. The figure shows mortality increases. The baseline parameters are the same as in Figure 3b. 
later in life. (See Almond et al. (2018) 's comprehensive review.) They point out that, while the initial fading out is consistent with the canonical Grossman (1972) model, the non-monotonicity of the effect is not. The Grossman model predicts a large immediate decline in the population's health after an in-utero shock that becomes hardly visible in adulthood (Figure 8a). Our model, by contrast, predicts exactly the U-shaped pattern described by Almond et al. (2018). Figure 8b documents that lowering initial health $\mu_{H}$ by $50 \%$ for the 1816 French cohort results in lower health among the survivors at all ages - both in levels and in percentages - with a u-shaped pattern in age. This occurs without complementary or compensating investments. The reason this happens in our model - but not in Grossman's - is that depreciation in our model is not multiplicative in the stock. ${ }^{32}$

These results also suggest it is not possible to identify the effects of in-utero shocks with health data for adolescents or young adults only. Schiman et al. (2017), who study the effects of experiencing WWII in utero and early childhood, find that its effects on health, disability, and employment among adults are not visible for young adults, but grow with age, as predicted here.

Mortality. Mortality at all ages also increases when initial conditions worsen but, interestingly, displays markedly different patterns depending on the metrics used. When measured in levels, the effects are again U-shaped. The intuition for this is simple. Among adolescents and young adults, the average level of health is high and very few individuals are close to the threshold, so shifting the distribution of health has very little impact on mortality. But shifts in the distribution will result in higher death rates as the distribution gets closer to the threshold at older ages. When expressed in percentage terms however, the predicted effects of negative in-utero shocks on mortality fall with age (Figure 8c), though this pattern is not necessarily monotonic: in middle ages, when mortality levels are low, the effects can rise and fall due to small samples. This occurs because the level of mortality is U-shaped with age also. An important implication of this exercise for the empirical literature is that the predictions for the dynamic effects of shocks on mortality are very sensitive to the functional form one chooses to study its effects.

\footnotetext{
${ }^{32}$ Dalgaard et al. (2019)'s model of health deficits also predicts that in-utero shocks will result in health gaps that increase with age starting in adulthood. But they do not model mortality or the effects of early childhood shocks on mortality before adulthood. Furthermore, our model predicts a u-shape pattern of effects rather than a monotonically increasing effect. This u-shape results from our having an early childhood period where investments move the distribution of health up.
} 


\subsection{Scarring Effects of Wars}

Wars have long-lasting detrimental health effects among survivors. Such "scarring" effects have been documented in at least 13 European countries after WWII. Compared to less exposed survivors, individuals who were more exposed to the war experienced worse economic and health outcomes several decades later (e.g. Kesternich et al., 2014, Havari and Peracchi, 2017). ${ }^{33}$ Figure 9a Panel a (reproduced from Wilson et al. 2014) shows the survival rates of World War I on New Zealand military personnel, comparing those that served in the war (1914 cohort) versus those that did not (1918 cohort).

The model successfully reproduces this survival pattern when the war is modeled as a temporary decrease in $I$. Figure $9 \mathrm{~b}$ shows the survival curve obtained from estimating a model with a 4-year decline in I at age 18 (corresponding to WWI and the 1918 flu pandemic) and a 6-year decline in $I$ at age 43 (corresponding to WWII), and simulating the counterfactual curve if the $I$-shock due to the war is eliminated. ${ }^{34}$ The model predicts what Wilson et al. (2014) observe in the data for their counterfactual (1900) cohort: the survival curve for the affected cohort is lower than the survival curve for the unaffected cohort, both during the war and after. The model predicts scarring: the mortality rates for survivors remain elevated throughout their lives relative to the counterfactual mortality that would have occurred in the absence of WWI. We estimate that WWI lowered life expectancy by approximately 16 years for the male 1896 cohort, and WWII lowered it by another 2 years. ${ }^{35}$

\footnotetext{
${ }^{33}$ Costa (2012) documents scarring effects of the American Civil War on surviving soldiers.

${ }^{34}$ This assumption is consistent with historical data for WWII. GDP declined substantially during the war and 20 to $55 \%$ of it was appropriated by Germans during the occupation (Occhino et al., 2007). Food rationing began in 1940. We can assume that the war is a different type of shock, but we do not obtain substantially better fit with these alternatives. Results available upon request.

${ }^{35}$ These estimates can be improved upon. We impose an equal annual shock during wars. The fit for this cohort can be improved substantially if we allow every year of the wars to have its own effect. But of course, this also lowers the degrees of freedom. Interestingly, if we do this, we find that 1914 was a particularly bad year, with investment estimated to be -5.3 that year, instead of the 0.57 annual investment we otherwise estimate for the cohort. The results are shown in Appendix table 5 .
} 
Figure 9: The effects of WWI on survival and mortality

(b) Effects of WWI and WWII among French men (a) Effect of WWI among New Zealand soldiers born in 1896
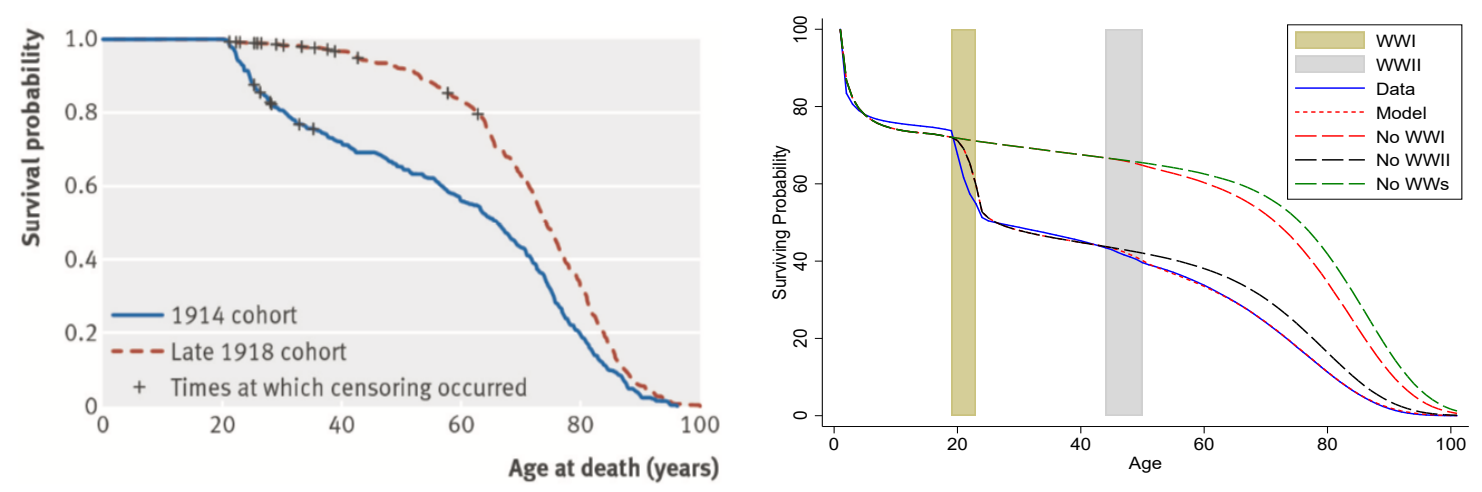

(c) Mortality rates French men born in 1896

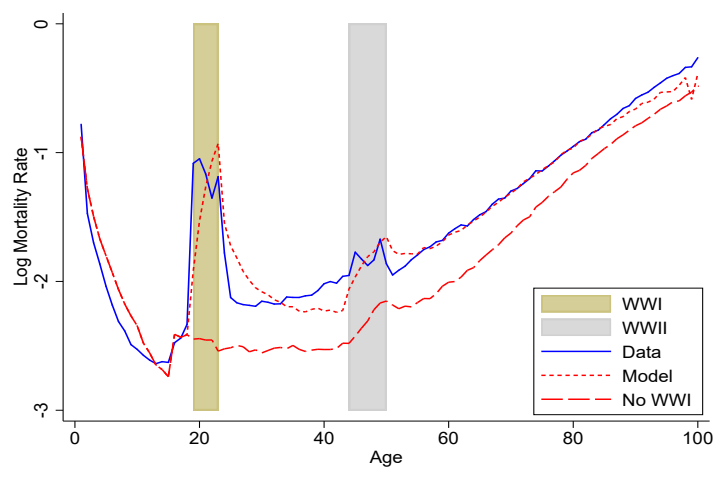

Note: Panel $a$ is from Wilson et al. (2014) and shows the scarring effect of WWI. It compares the survival curve of the cohort of young New Zealand military personnel who left for the war in 1914, with the cohort who left for the war in 1918 but did not see active combat. The median age in the sample is 24 . Panel $b$ shows the scarring effect of WWI for men born in France in 1896 who turned 18 when WWI started in 1914 and who would have served in the military. The model for this cohort includes one more parameter for WWI and another for WWII: we allow for $I$ to be different during each war. Instead of using a comparison group, the model is used to compute counterfactual curves showing what survival profiles would look like in the absence of either or both wars. Panel $c$ shows the mortality rates by age for the 1986 cohort of French men. 


\subsection{Harvesting Effects}

Extreme weather or pollution events appear to displace the distribution of deaths in the short term, creating a sudden increase in the number of deaths followed by abnormally low mortality. In demography, this phenomenon is known as "harvesting" and has been, for instance, documented in France during the 2003 heatwave, as shown in Figure 10a reproduced from Toulemon and Barbieri (2008). ${ }^{36}$

Suppose that the death threshold $\underline{H}$ is mostly a function of the environment. Figure 10b shows the simulated effect of a temporary increase in the threshold at ages 60 and 61 on the mortality of the 1816 cohort. It results in very high mortality during the shock. But mortality starts dropping before the shock ends because the frailest individuals have already died in the first period of the shock, so later on only those that receive a large negative idiosyncratic shock die. Once the weather disruption ends, and the threshold is restored to its original (lower) level, mortality falls substantially because there are very few individuals close to the new (lower) threshold. This holds true for a long time until the aging process naturally lowers health stocks again, closer to the new lower threshold. Thus a change in the death threshold generates harvesting, and does so by killing the least healthy individuals of the cohort. A key characteristic of a threshold change is that it does not affect the health of the living. ${ }^{37}$

Heat waves and other forms of bad weather also generate excess mortality among children (Figure 10c). However, the displacement effect is substantially more spread out among children. In other words, the children who die as a result of the bad weather would not be dying immediately right after the bad weather ends - they would be living substantially longer lives. Thus the cost of this event is much larger in terms of life expectancy when it affects children than the elderly. This occurs because, among children, investment levels are high relative to depreciation and mortality rates are falling. In contrast, depreciation among the elderly is much larger.

\footnotetext{
${ }^{36}$ See Schwartz (2000) or Zeger et al. (1999) for the effects of pollution, and Deschenes and Moretti (2009) or Deschênes and Greenstone (2011) for the effects of very hot or very cold weather.

${ }^{37}$ It is unclear whether weather shocks have no effects on the health of those that do not die. See Deschenes and Moretti (2009) or Deschênes and Greenstone (2011) for discussions of this.
} 
Figure 10: The effects of temporary increases in the threshold

(a) Harvesting during the French 2003 Heatwave

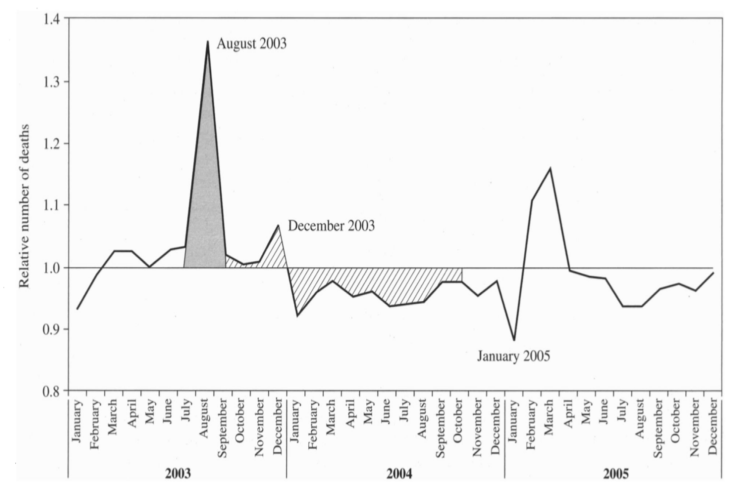

(b) Harvesting in the model among the old

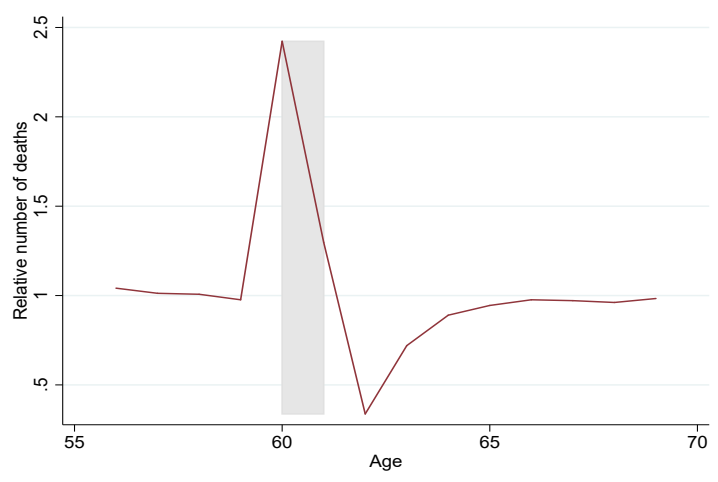

(c) Harvesting in the model among the young

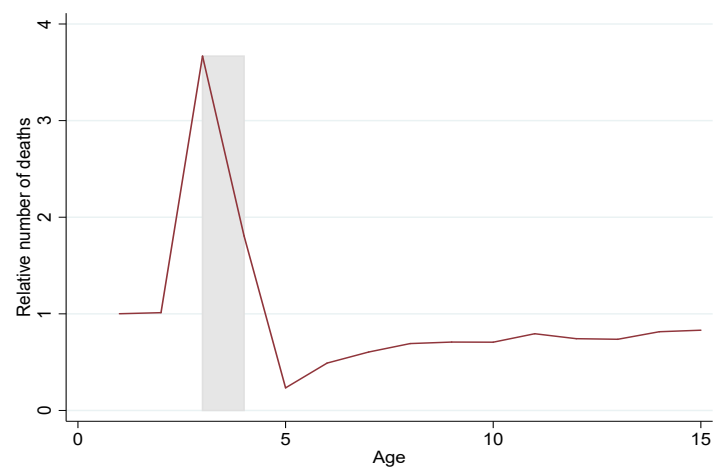

Note: Panel $a$ is is from Toulemon and Barbieri (2008) and shows the mortality displacement created by the French 2003 Heatwave. The number of excess deaths in Summer 2003 is computed relative to the number of deaths during the same period in 2000 . The grey (hatched) area corresponds to an excess (deficit) of 15,000 deaths. These excess deaths are computed for the entire population. Panel $b$ shows the simulated effects of a temporary increase in the threshold (from 0 to 0.8 ) at ages 60 and 61 on the 1816 French cohort (setting the accident rate to 0 for simplicity) which results in approximately 8000 excess deaths during the shock and fewer deaths for the subsequent 2 years. Panel $c$ shows the simulated effects of a temporary increase in the threshold (from 0 to 0.8 ) at ages 3 and 4 on the 1816 French cohort (setting the accident rate to 0 for simplicity) which results in approximately 40,000 excess deaths during the shock. The effect is much larger among the young because many more children are close to the threshold as shown in Figure 3a. 
Figure 11: Effects of temporary shocks on log mortality rates
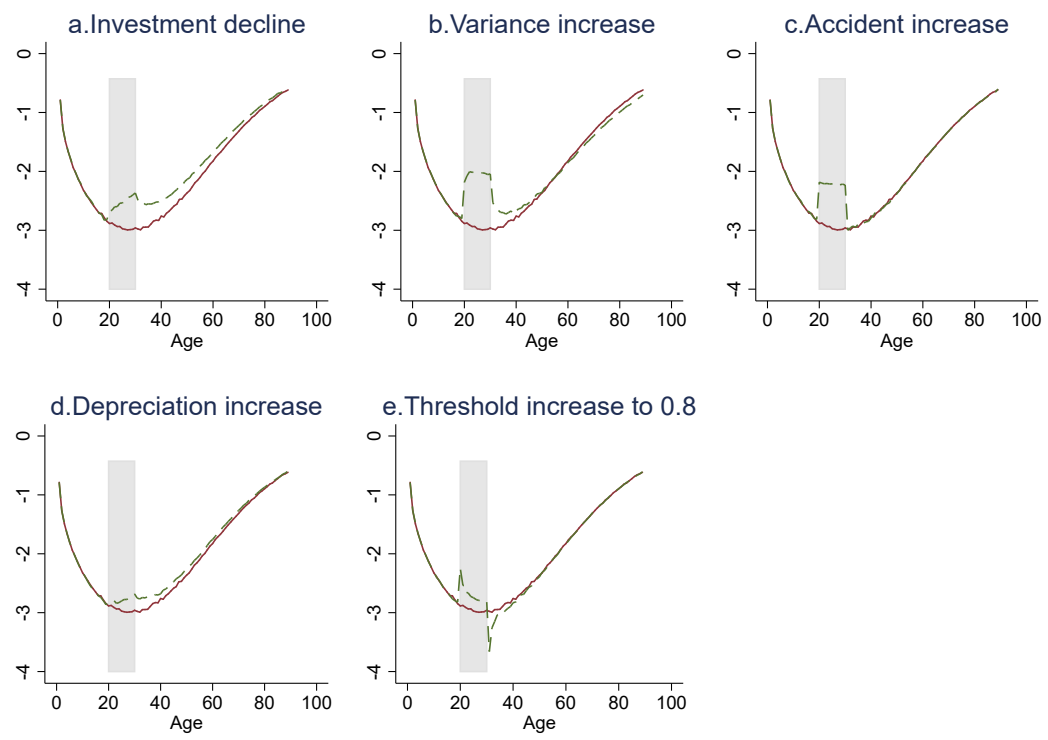

Note: Results from simulations using the 1816 cohort parameters and assuming no adolescent hump. Shocks correspond to a $50 \%$ change in the parameter, except for the threshold, which is assumed to increase to 0.8 from 0 . The shock starts at age 20 and lasts 10 years, ending at age 30 .

\subsection{Other types of shocks}

The previous two sections illustrate the effects of temporary decreases in $I$ or increases in the threshold, but do not compare their effects in the same scale because we aimed to reproduced existing published results. Figure 11 shows how log mortality rates respond to all types of temporary shocks. Each shock leaves a unique imprint on mortality. Temporary investment and depreciation decreases have similar scarring effects: mortality rises when the shock starts and then starts falling after the shock ends but it does not return to its counterfactual level. On the other hand, only changes in the threshold generate harvesting. Only variance changes results in a "cross over" in mortality rates in old ages. And only accident increases leave mortality rates unchanged once the shock ends. Appendix Figure 20 further reveals that the pattern of these responses over time is not the same when viewed in logs or in levels.

There are some important patterns that cannot be rationalized with these simple shocks. For example, the literature has shown that recessions lower mortality 
in the short term but increase it in the long term. To generate this type of pattern, one would need to assume that recessions affect more than one parameter and consider combinations of possibly asynchronous shocks. For example, it could be that during a recession accidents fall in the short term but lifetime incomes are permanetly decreased as hypothesized by Schwandt and Von Wachter (2019).

\section{Implications for optimal investments}

\subsection{Optimization in a stationary environment}

So far we have considered a population that receives constant investments in its health, uniformly over the lifetime. But is that behavior a reasonable approximation if resources are optimally allocated over the lifetime? To answer this question, this section relaxes the simplifying assumption of constant investment, and estimates the optimal investment profile that a social planner concerned with maximizing the life-expectancy of a population would choose. Remarkably, while this optimal investment profile indeed deviates from the constant investment rule studied in the previous sections, it would result in very similar patterns of mortality. In other words, the optimal investment sequence does not fundamentally change the age-profile of mortality rates. We then evaluate the life expectancy gains resulting from optimization.

First we develop notation to describe the problem that a benevolent social planner would face. We solve this problem under two key assumptions. The first key assumption is that the planner has a fixed budget but has the ability to borrow and save costlessly - in other words, the planner knows exactly what the total lifetime resources are for a given cohort and can be redistribute these resources across the lifetime at no cost. ${ }^{38}$ The second assumption we make is that the planner wishes to maximize life expectancy.

The survival function tracks the probability of surviving over time. It is naturally expressed as a function of the cdf of health in the population. The probability of surviving until the end of period $a$ is $S_{a}=1-F_{a}(0)$. Life expectancy at birth for

\footnotetext{
${ }^{38}$ This is a standard set of assumptions in this type of models, for example see Murphy and Topel (2006).
} 
a given cohort is conveniently related to the survival function

$$
L E=\sum_{a=1}^{\infty} S_{a}
$$

Several observations are in order. First, in practice, this is a finite sum. Second, this is the cohort's life expectancy, not the "period" life expectancy which is usually reported. The social planner now chooses an investment path $\mathcal{I}=\left\{I_{a}\right\}_{a \in \mathbb{N}}$ that is age-dependent, instead of keeping the investment level $I$ constant over the lifetime. The planner can move resources over time periods costlessly, as if a perfect annuity were available, and faces a given lifetime budget, $B$. Then the optimization problem takes the form

$$
\begin{array}{cc}
\max _{\mathcal{I}} L E(\mathcal{I})= & \max _{\left\{I_{a}\right\}} \sum_{a=1}^{\infty} S_{a}(\mathcal{I}) \\
\text { s.t. } & \sum_{a=1}^{\infty} I_{a} \cdot S_{a}(\mathcal{I}) \leq B
\end{array}
$$

The social planner chooses an optimal path such that the marginal effect of increasing investment at a given age is equalized across all ages. The first order conditions are given by

$$
\sum_{s=a}^{\infty} \frac{\partial S_{s}(\mathcal{I})}{\partial I_{a}}-\lambda\left[S_{a}(\mathcal{I})+\sum_{s \geq a}^{\infty} I_{s} \frac{\partial S_{s}(\mathcal{I})}{\partial I_{a}}\right]=0, \forall a>0
$$

where $\lambda$ is the Lagrange multiplier and therefore $\frac{1}{\lambda}$ represents the shadow cost for the social planner, starting from the optimal path, of an additional year of life expectancy. Both terms in the bracket are positive, illustrating the key dynamic tradeoff in investment with a fixed budget. An additional investment at one age increases the number of survivors at all subsequent ages, exerting greater pressure on the budget at all subsequent periods. Intuitively, this channel gets weaker and weaker at older ages because mortality rates are high at old ages even with investments. While we were unable to formally makes this point analytically, we show numerically that this intuition is valid in the range of parameters estimated from the data. 


\subsection{Timing of optimal investments, polynomials}

To estimate the optimal investment, we follow a lower-dimensional sieves estimation method. ${ }^{39}$ We start by approximating the investment profile over age with a first order function of age (adding 2 parameters) and then with a second order polynomial ( 3 more parameters). We impose the constraint that the total spending per cohort is the same as the budget resulting from our estimated constant lifetime investment i.e. $B=\sum_{a=1}^{100} \hat{I} \cdot S_{a}(\mathcal{I})$. Given budget $B$ we run a grid search to find the quadratic investment profile that maximizes the life expectancy of the cohort.

The results of this exercise are displayed in Figure 12. Relative to the case with a constant function, an optimal linear investment function redistributes more resources to the young. If we allow a quadratic term then we find that a U-shape investment profile is optimal to maximize the average life-expectancy in the population (panel a). Our original model sets $I$ to be constant in levels. But in percentage terms, relative to the baseline level of health at a given age, $I$ was already U-shaped in the basic model. What we find then is that the optimal investment is even more U-shaped - it transfers additional resources to the young and the old, away from the middle-aged individuals.

These results show that optimal health investments are largest when health is at its lowest - that is, at very young and very old ages. Interestingly, health care expenditures by age in most countries actually follow this age-profile (Alemayehu and Warner, 2004). These findings are also consistent with empirical findings which show that health and the demand for medical services are negatively correlated (Wagstaff, 1986) and that medical expenditures rise sharply with age (e.g. De Nardi et al. 2010). ${ }^{40}$

Panel $b$ shows the mortality curves before and after optimization - they have the same basic shape we have observed. Yhe resulting survival curves are flatter in adulthood and steeper in old ages, suggesting the rectangularization of survival

\footnotetext{
${ }^{39} \mathrm{~A}$ fully nonparametric approach for the optimal investment profile over the lifetime would require optimizing over a hundred or so parameters (one for each age) for each cohort. In the absence of a closed-form solution, this is impractical. It is also not feasible since we have 100 data points: if we allow for a unique investment level at every age we are under-identified (we would have 100 data points and at least 106 parameters to estimate).

${ }^{40}$ These results are in contrast with the predictions of the Grossman model which predicts that investments would decline with age as individuals near death. See Wagstaff (1986)for an early discussion, or Strulik (2015) for a more recent discussion of this issue.
} 
Figure 12: Optimal Investment Levels by Age
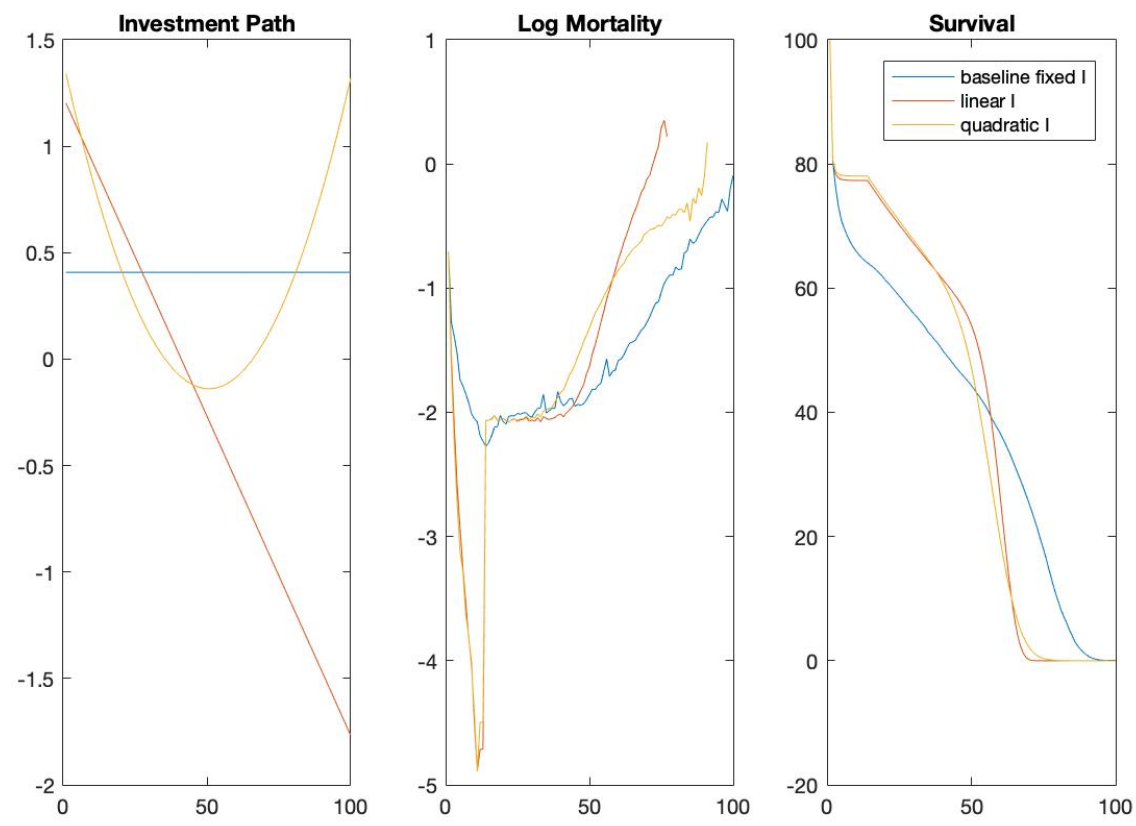

Note: The first panel represents the estimated investment path when investment is constrained to be constant (blue line), linear (red line), or quadratic (yellow). In the second and third panel, 1816 cohort data is represented in blue. Both linear and quadratic optimal investment paths would devote more resources to younger cohorts, reducing mortality rates in the early years.

might be in part associated with the emergence of optimal investments. Optimizing investment results in a gain of about 3 years of life expectancy in the specific case we show in Figure 12, based on the estimated parameters for French women born in 1816.

Optimization when budgets depend on health. We have solved the optimization problem under the assumption that stock of available resources is not influenced by the health of the population. But if food and other resources are produced rather than taken from the environment, health is likely to impact resources by affecting the work capacity of the population. Indeed, nutrition levels and disease rates have been shown to affect productivity and wages (Thomas et al., 2004). They also affect inputs into wages such as cognition and education (Field et al., 2009). Many empirical studies report a correlation between income and health (Cutler et al., 2012, Chetty et al., 2016) as noted above. While our baseline model embeds the 
effect of resources on health, a causal link going in the other direction is also likely at play: people who get sick or are hospitalized suffer a subsequent drop in income (Smith, 1999, Dobkin et al., 2018). With panel data on wages, it would be possible to improve on our estimates to account for these effects.

Overlapping generations. Another natural extension would be to embed our model in an overlapping generations setting to reflect the fact that most social insurace programs, including health care insurance, involve transfers across cohorts at a given point in time, rather than within-cohort transfers over time (as we have considered here for simplicity). An overlapping generation model could also be used to link the health of the parents with that of their children, a mechanism that has found some support in the empirical literature.

\section{Conclusion}

This paper proposes a parsimonious production function to study the evolution of health and mortality over the life course of a population with heterogeneous health endowments. The basic model can be easily estimated by using observed cohort mortality rates. Despite its simplicity, this model tracks the evolution of the mortality profile of human cohorts born 1816 to 1940 as well as other species, and it can explain many important mortality patterns documented in the literature, including the rectangularization of survival curves and SES gradients in health. We also show how to use the model to understand the dynamic treatment effects of in-utero shocks and other temporary shocks like wars.

The parsimony of the model relies on transparent but strong parametric assumptions. In particular, we assumed that the environment is stable and exogenously provides a constant level of resources. This is reasonable for primates or early human populations before the rise of modern medicine and other technical innovations, but not for contemporary human populations. We have explored how to incorporate changes in the environment into the model and shown these changes can qualitatively produce many patterns in the existing literature. We also assume that health shocks are i.i.d and normally distributed. We argue that these assumptions are roughly consistent with the evidence in the literature and with the patterns observed in the HMD data. Alternative assumptions for this distribution of annual shocks could be further investigated to integrate the mortality impact of 
contagious diseases such as the COVID-19 pandemic.

The model can only be expanded further to consider the role of behavior and policy. At this stage, our analysis suggests that, in the absence of financial frictions, optimal health expenditures are U-shaped over the lifetime in this model. With systematic data on health inputs and shocks, as well as prices and budgets over the life course, these implications could be fruitfully explored further. The model can be used to investigate other interesting questions such as the origins of gender or cross-country differences. We leave these to future research. 


\section{References}

Alemayehu, Berhanu and Kenneth E. Warner, "The lifetime distribution of health care costs," Health services research, 2004, 39 (3), 627-642.

Almond, Douglas and Janet Currie, "Killing me softly: The fetal origins hypothesis," The Journal of Economic Perspectives, 2011, 25 (3), 153-172.

_ , , , and Valentina Duque, "Childhood circumstances and adult outcomes: Act II," Journal of Economic Literature, 2018, 56 (4), 1360-1446.

Armitage, Peter and Richard Doll, "The age distribution of cancer and a multistage theory of carcinogenesis," British journal of cancer, 1954, 8 (1), 1.

Behringer, Verena, Tobias Deschner, Caroline Deimel, Jereon MG Stevens, and Gottfried Hohmann, "Age-related changes in urinary testosterone levels suggest differences in puberty onset and divergent life history strategies in bonobos and chimpanzees," Hormones and behavior, 2014, 66 (3), 525-533.

Benyamini, Yael and Ellen L Idler, "Community studies reporting association between self-rated health and mortality: additional studies, 1995 to 1998," Research on aging, 1999, 21 (3), 392-401.

Bharadwaj, Prashant, Katrine Vellesen Loken, and Christopher Neilson, "Early life health interventions and academic achievement," American Economic Review, 2013, 103 (5), 1862-91.

Bronikowski, Anne M., Jeanne Altmann, Diane K. Brockman, Marina Cords, Linda M. Fedigan, Anne Pusey, Tara Stoinski, William F. Morris, Karen B. Strier, and Susan C. Alberts, "Aging in the natural world: comparative data reveal similar mortality patterns across primates," Science, 2011, 331 (6022), 13251328.

Case, Anne and Angus S Deaton, "Broken down by work and sex: How our health declines," in "Analyses in the Economics of Aging," University of Chicago Press, 2005, pp. 185-212. 
- , Darren Lubotsky, and Christina Paxson, "Economic status and health in childhood: The origins of the gradient," The American Economic Review, 2002, 92 (5), $1308-1334$.

Centers for Disease Control and Prevention, "Summary health statistics: National health interview survey," Atlanta, GA: Centers for Disease Control and Prevention, 2014.

Chetty, Raj, Michael Stepner, Sarah Abraham, Shelby Lin, Benjamin Scuderi, Nicholas Turner, Augustin Bergeron, and David Cutler, "The association between income and life expectancy in the United States, 2001-2014," Jama, 2016, 315 (16), 1750-1766.

Costa, Dora L, "Scarring and mortality selection among Civil War POWs: A longterm mortality, morbidity, and socioeconomic follow-up," Demography, 2012, 49 (4), 1185-1206.

Crimmins, Eileen M, "Socioeconomic differentials in mortality and health at the older ages," Genus, 2005, pp. 163-176.

Cullen, Mark R, Michael Baiocchi, Karen Eggleston, Pooja Loftus, and Victor Fuchs, "The weaker sex? Vulnerable men and women's resilience to socioeconomic disadvantage," SSM-population health, 2016, 2, 512-524.

Currie, Janet and Mark Stabile, "Socioeconomic Status and Child Health: Why Is the Relationship Stronger for Older Children?," The American Economic Review, 2003, 93 (5), 1813-1823.

Cutler, David, Angus Deaton, and Adriana Lleras-Muney, "The determinants of mortality," The Journal of Economic Perspectives, 2006, 20 (3), 97-120.

Cutler, David M., Adriana Lleras-Muney, Tom Vogl, S. Glied, and P. C. Smith, "Socioeconomic Status and Health: Dimensions and Mechanisms.," in "The Oxford Handbook of Health Economics," Oxford University Press, 2012.

Cutler, David M and Adriana Lleras-Muney, "Understanding differences in health behaviors by education," Journal of health economics, 2010, 29 (1), 1-28. 
Dalgaard, Carl-Johan and Holger Strulik, "Optimal aging and death: understanding the Preston curve," Journal of the European Economic Association, 2014, 12 (3), 672-701.

- , Casper Worm Hansen, and Holger Strulik, "Accounting for fetal origins: Health capital vs. health deficits," Health Deficits (October 19, 2019), 2019.

de La Rochebrochard, Elise, "Age at puberty of girls and boys in France: Measurements from a survey on adolescent sexuality," Population: An English Selection, 2000, pp. 51-79.

Deaton, Angus S. and Christina H. Paxson, "Aging and inequality in income and health," The American Economic Review, 1998, 88 (2), 248-253.

den Berg, Gerard J Van, Gabriele Doblhammer, and Kaare Christensen, "Exogenous determinants of early-life conditions, and mortality later in life," Social science \& medicine, 2009, 68 (9), 1591-1598.

_ , Maarten Lindeboom, and France Portrait, "Economic conditions early in life and individual mortality," American Economic Review, 2006, 96 (1), 290-302.

Deschenes, Olivier and Enrico Moretti, "Extreme weather events, mortality, and migration," The Review of Economics and Statistics, 2009, 91 (4), 659-681.

Deschênes, Olivier and Michael Greenstone, "Climate change, mortality, and adaptation: Evidence from annual fluctuations in weather in the US," American Economic Journal: Applied Economics, 2011, 3 (4), 152-85.

Dobkin, Carlos, Amy Finkelstein, Raymond Kluender, and Matthew J Notowidigdo, "The economic consequences of hospital admissions," American Economic Review, 2018, 108 (2), 308-52.

Field, Erica, Omar Robles, and Maximo Torero, "Iodine deficiency and schooling attainment in Tanzania," American Economic Journal: Applied Economics, 2009, 1 (4), 140-169.

Fogel, Robert W., "Economic Growth, Population Theory, and Physiology: The Bearing of Long-Term Processes on the Making of Economic Policy," The American Economic Review, 1994, 84 (3), 369-395. 
Galama, Titus J and Hans Van Kippersluis, "A Theory of Socio-economic Disparities in Health over the Life Cycle," The Economic Journal, 2018, 129 (617), 338-374.

Gavrilov, Leonid Anatolevich and Natalia Sergeevna Gavrilova, The biology of life span: a quantitative approach., Harwood Academic Publisher New York, 1991.

Goldin, Claudia and Adriana Lleras-Muney, " $X X>X Y$ ?: The changing female advantage in life expectancy," Journal of health economics, 2019, 67, 102224.

Gompertz, Benjamin, "On the nature of the function expressive of the law of human mortality, and on a new mode of determining the value of life contingencies," Philosophical transactions of the Royal Society of London, 1825, 115, 513-583.

Grossman, Michael, "On the concept of health capital and the demand for health," Journal of Political economy, 1972, 80 (2), 223-255.

Halliday, Mark H, Alessandra N Garcia, Anita B Amorim, Gustavo C Machado, Jill A Hayden, Evangelos Pappas, Paulo H Ferreira, and Mark J Hancock, "Treatment effect sizes of mechanical diagnosis and therapy for pain and disability in patients with low back pain: a systematic review," journal of orthopaedic E sports physical therapy, 2019, 49 (4), 219-229.

Halliday, Timothy, "Health inequality over the life-cycle," The BE Journal of Economic Analysis \& Policy, 2011, 11 (3).

_ , Bhashkar Mazumder, and Ashley Wong, "Intergenerational health mobility in the US," 2018.

Havari, Enkelejda and Franco Peracchi, "Growing up in wartime: Evidence from the era of two world wars," Economics \& Human Biology, 2017, 25, 9-32.

Heligman, Larry and John H. Pollard, "The age pattern of mortality," Journal of the Institute of Actuaries, 1980, 107 (01), 49-80.

House, James S, Paula M Lantz, and Pamela Herd, "Continuity and change in the social stratification of aging and health over the life course: evidence from a nationally representative longitudinal study from 1986 to 2001/2002 (Americans' Changing Lives Study)," The Journals of Gerontology Series B: Psychological Sciences and Social Sciences, 2005, 60 (Special_Issue_2), S15-S26. 
Human Mortality Database. University of California, Berkeley (USA), and Max Planck Institute for Demographic Research (Germany). Available at www. mortality.org. Data downloaded in August 2017.

Hummer, Robert A. and Joseph T. Lariscy, "Educational attainment and adult mortality," in "International handbook of adult mortality," Springer, 2011, pp. 241-261.

Kaestner, Robert, Cuiping Schiman, and Jason Ward, "Education and health over the life cycle," Economics of Education Review, 2020, 76, 101982.

Kesternich, Iris, Bettina Siflinger, James P Smith, and Joachim K Winter, "The effects of World War II on economic and health outcomes across Europe," Review of Economics and Statistics, 2014, 96 (1), 103-118.

Lando, David, Credit Risk Modeling: Theory and Applications, Princeton University Press, 2004.

Li, Ting and James Anderson, "Shaping human mortality patterns through intrinsic and extrinsic vitality processes," Demographic research, 2013, 28, 341-372.

Limpert, Eckhard, Werner A. Stahel, and Markus Abbt, "Log-normal distributions across the sciences: Keys and clues on the charms of statistics, and how mechanical models resembling gambling machines offer a link to a handy way to characterize log-normal distributions, which can provide deeper insight into variability and probability—normal or log-normal: That is the question," BioScience, 2001, 51 (5), 341-352.

Lindeboom, Maarten, France Portrait, and Gerard J Van den Berg, "Long-run effects on longevity of a nutritional shock early in life: the Dutch Potato famine of 1846-1847," Journal of health economics, 2010, 29 (5), 617-629.

Liu, Zuyun, Brian H Chen, Themistocles L Assimes, Luigi Ferrucci, Steve Horvath, and Morgan E Levine, "The Role of Epigenetic Aging in Education and Racial/Ethnic Mortality Disparities Among Older US Women," Psychoneuroendocrinology, 2019.

Loudon, Irvine, "Maternal mortality: 1880-1950. Some regional and international comparisons," Social history of medicine, 1988, 1 (2), 183-228. 
Lynch, Scott M, "Cohort and life-course patterns in the relationship between education and health: A hierarchical approach," Demography, 2003, 40 (2), 309-331.

Masters, Ryan K, "Economic Conditions in Early Life and Circulatory Disease Mortality," Population and Development Review, 2018, 44 (3), 519-553.

McGee, Daniel L, Youlian Liao, Guichan Cao, and Richard S Cooper, "Selfreported health status and mortality in a multiethnic US cohort," American journal of epidemiology, 1999, 149 (1), 41-46.

Murphy, Kevin M. and Robert H. Topel, "The value of health and longevity," Journal of political Economy, 2006, 114 (5), 871-904.

Nardi, Mariacristina De, Eric French, and John B Jones, "Why do the elderly save? The role of medical expenses," Journal of political economy, 2010, 118 (1), 39-75.

Occhino, Filippo, Kim Oosterlinck, and Eugene N White, "How occupied France financed its own exploitation in World War II," American Economic Review, 2007, 97 (2), 295-299.

Palloni, Alberto and Hiram Beltrán-Sánchez, "Demographic Consequences of Barker Frailty," in "Dynamic Demographic Analysis," Springer, 2016, pp. 147176.

Pinker, Steven, The better angels of our nature: Why violence has declined, Vol. 75, Viking New York, 2011.

Pompei, Francesco and Richard Wilson, "A quantitative model of cellular senescence influence on cancer and longevity," Toxicology and industrial health, 2002, 18 (8), 365-376.

Preston, Samuel $\mathbf{H}$ and Etienne Van de Walle, "Urban French mortality in the nineteenth century," Population studies, 1978, 32 (2), 275-297.

Preston, Samuel, Patrick Heuveline, and Michel Guillot, Demography: measuring and modeling population processes, Oxford: Blackwell Publishers, 2000. 
Rosenberg, Karen and Wenda Trevathan, "Bipedalism and human birth: The obstetrical dilemma revisited," Evolutionary Anthropology: Issues, News, and Reviews, 1995, 4 (5), 161-168.

Rosenberg, Karen R, "The evolution of modern human childbirth," American Journal of Physical Anthropology, 1992, 35 (S15), 89-124.

Ross, Catherine E and Chia ling Wu, "The links between education and health," American sociological review, 1995, pp. 719-745.

Schiman, Jeffrey C, Robert Kaestner, and Anthony T Lo Sasso, "Early Childhood Health Shocks and Adult Wellbeing: Evidence from Wartime Britain," Technical Report, National Bureau of Economic Research 2017.

Schwandt, Hannes and Till Von Wachter, "Unlucky cohorts: Estimating the longterm effects of entering the labor market in a recession in large cross-sectional data sets," Journal of Labor Economics, 2019, 37 (S1), S161-S198.

Schwartz, Joel, "Harvesting and long term exposure effects in the relation between air pollution and mortality," American journal of epidemiology, 2000, 151 (5), 440448.

Sharrow, David J. and James J. Anderson, "Quantifying Intrinsic and Extrinsic Contributions to Human Longevity: Application of a Two-Process Vitality Model to the Human Mortality Database," Demography, 2016, 53 (6), 2105-2119.

Smith, James P., "Healthy bodies and thick wallets: the dual relation between health and economic status," The journal of economic perspectives: a journal of the American Economic Association, 1999, 13 (2), 144.

Steves, Claire Joanne, Timothy D Spector, and Stephen HD Jackson, "Ageing, genes, environment and epigenetics: what twin studies tell us now, and in the future," Age and ageing, 2012, 41 (5), 581-586.

Strulik, Holger, "A Closed-form Solution for the Health Capital Model," Journal of Demographic Economics, 2015, 81 (3), 301-316.

Tanner, James Mourilyan and James Mourilyan Tanner, A history of the study of human growth, Cambridge University Press, 1981. 
Thiele, Thorvald Nicolai, "On a mathematical formula to express the rate of mortality throughout the whole of life, tested by a series of observations made use of by the Danish Life Insurance Company of 1871," Journal of the Institute of Actuaries, 1871, 16 (5), 313-329.

Thomas, Duncan, Elizabeth Frankenberg, Jed Friedman, Jean-Pierre Habicht, Nathan Jones, Christopher McKelvey, Gretel Pelto, Bondan Sikoki, James P. Smith, Cecep Sumantri, and others, "Causal effect of health on labor market outcomes: Evidence from a random assignment iron supplementation intervention," California Center for Population Research, 2004.

Toulemon, Laurent and Magali Barbieri, "The mortality impact of the August 2003 heat wave in France: investigating the ?harvesting?effect and other longterm consequences," Population studies, 2008, 62 (1), 39-53.

Vaupel, James W., Kenneth G. Manton, and Eric Stallard, "The impact of heterogeneity in individual frailty on the dynamics of mortality," Demography, 1979, 16 (3), 439-454.

Wagstaff, Adam, "The demand for health: some new empirical evidence," Journal of Health economics, 1986, 5 (3), 195-233.

Weibull, Waloddi, "Wide applicability," Journal of applied mechanics, 1951, 103 (730), 293-297.

Wilcox, Allen J. and Ian T Russell, "Birthweight and perinatal mortality: I. On the frequency distribution of birthweight," International Journal of Epidemiology, 1983, 12 (3), 314-318.

Wilson, Nick, Christine Clement, Jennifer A Summers, John Bannister, and Glyn Harper, "Mortality of first world war military personnel: comparison of two military cohorts," Bmj, 2014, 349, g7168.

Wong, Chloe Chung Yi, Avshalom Caspi, Benjamin Williams, Ian W Craig, Renate Houts, Antony Ambler, Terrie E Moffitt, and Jonathan Mill, "A longitudinal study of epigenetic variation in twins," Epigenetics, 2010, 5 (6), 516-526.

Zeger, Scott L, Francesca Dominici, and Jonathan Samet, "Harvesting-resistant estimates of air pollution effects on mortality," Epidemiology, 1999, pp. 171-175. 


\section{Appendix Material Not For Publication}

This Appendix contains three parts

1. Appendix A contains additional Figures (A1) and Tables (A2)

2. Appendix B contains the proof that in the model everyone dies

3. Appendix $\mathrm{C}$ contains details of the estimation 


\section{Appendix A1: Figures}

Figure 13: Comparison of q-rate in the paper and HMD (1816)
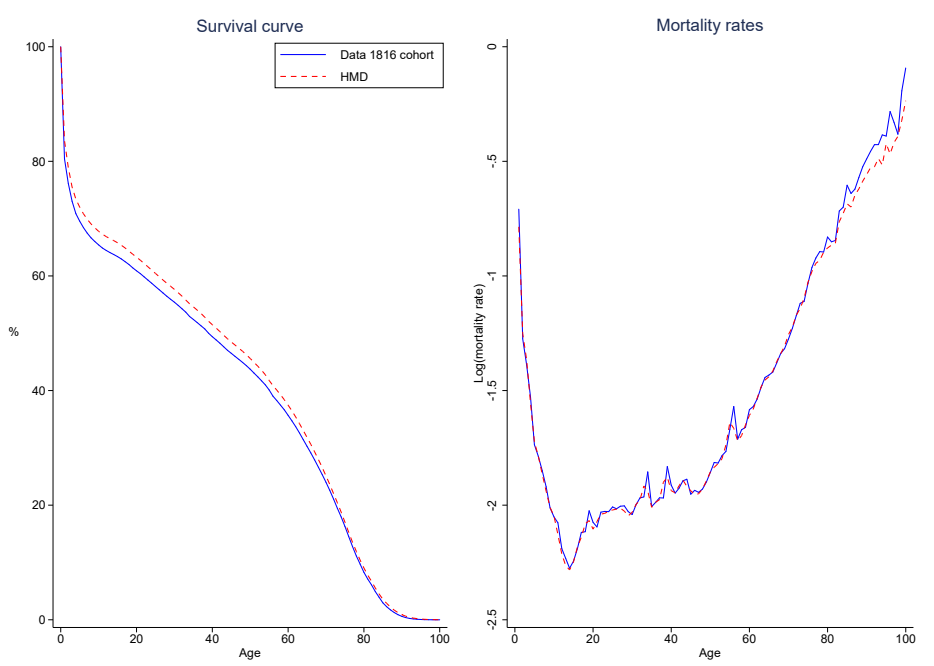

Note: The life expectancy is 38.25 years with the $q$ we use, to be compared with 39.86 with the $q$ in HMD and 39.83 years for the life expectancy computed by the HMD itself following a more involved statistical methodology. 
Figure 14: Cohort vs. Period statistics, French Women 1860 and 1940

(a) Cohort vs Period Life Expectancy for French Females

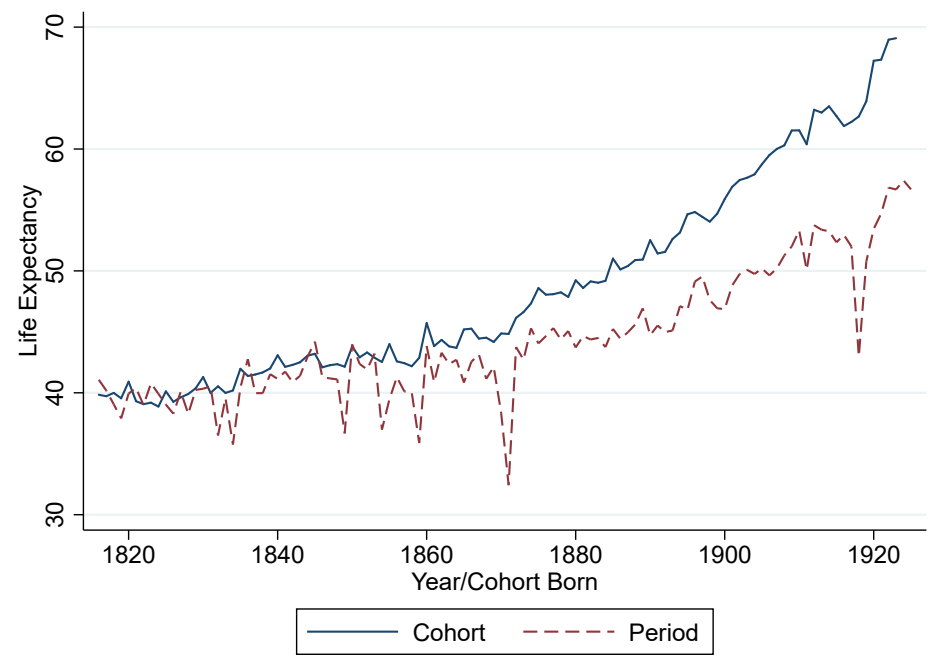

(b) Cohort vs Period Life Expectancy for French Males

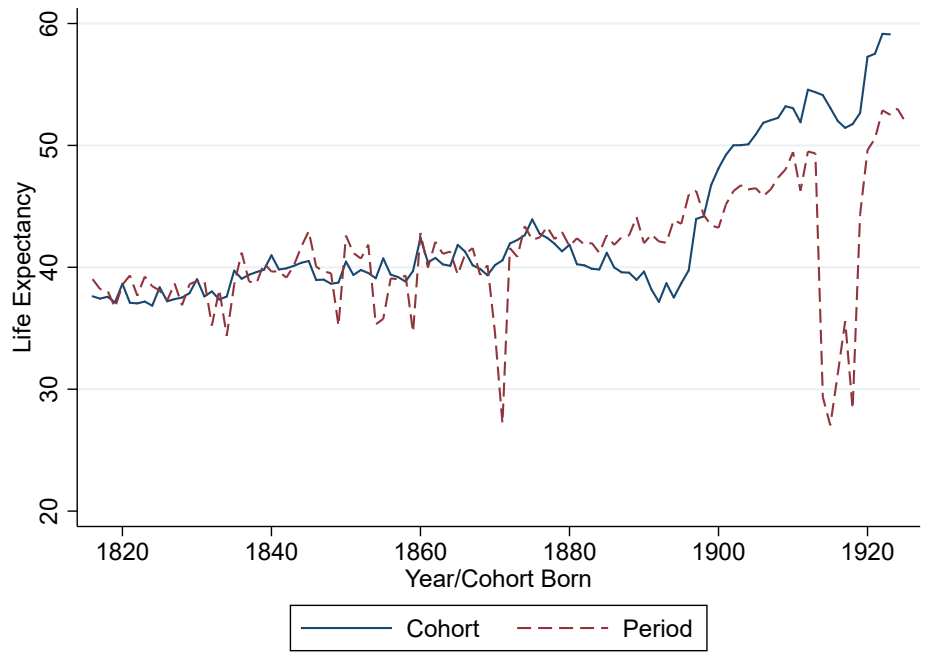

Note: Data from the Human Mortality Database. Panel a shows period and cohort mortality rates were almost identical for the cohort born in 1860, suggesting that for these cohorts the assumption of stationarity holds. In other words, the mortality rate at age 50 of a French woman born in 1860 is about the same as the one of a French woman who is 50 year old in 1860. In 1940 a large gap has appeared and the cohort mortality rates is significantly lower than the period rate. Panel $b$ shows the period and cohort life expectancy of French women since 1816. The two series are almost the same up to roughly 1860 and they diverge after, with the cohort life expectancy exceeding the period life expectancy substantially byt the end of the period. 
Figure 15: Age profile of mortality of women born in France between 1860 and 1940, by decade Log-Mortality Rates. French Women cohorts bom between 1860 and 1939 (Source:HMD)
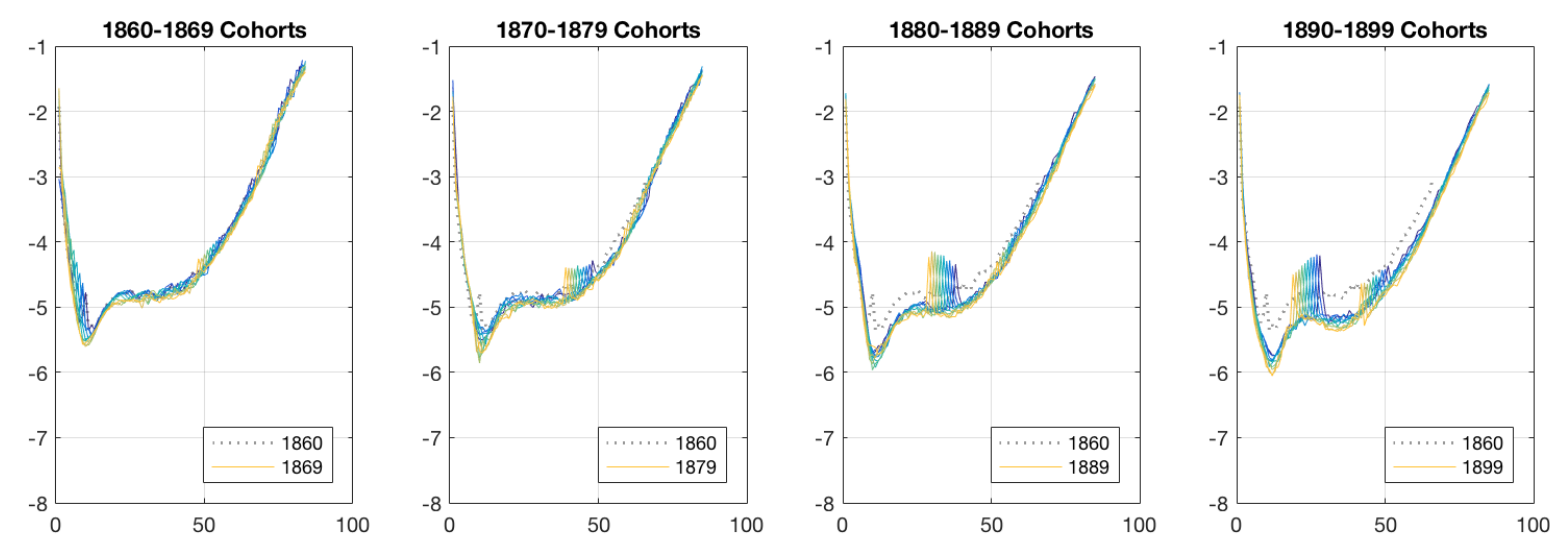

to
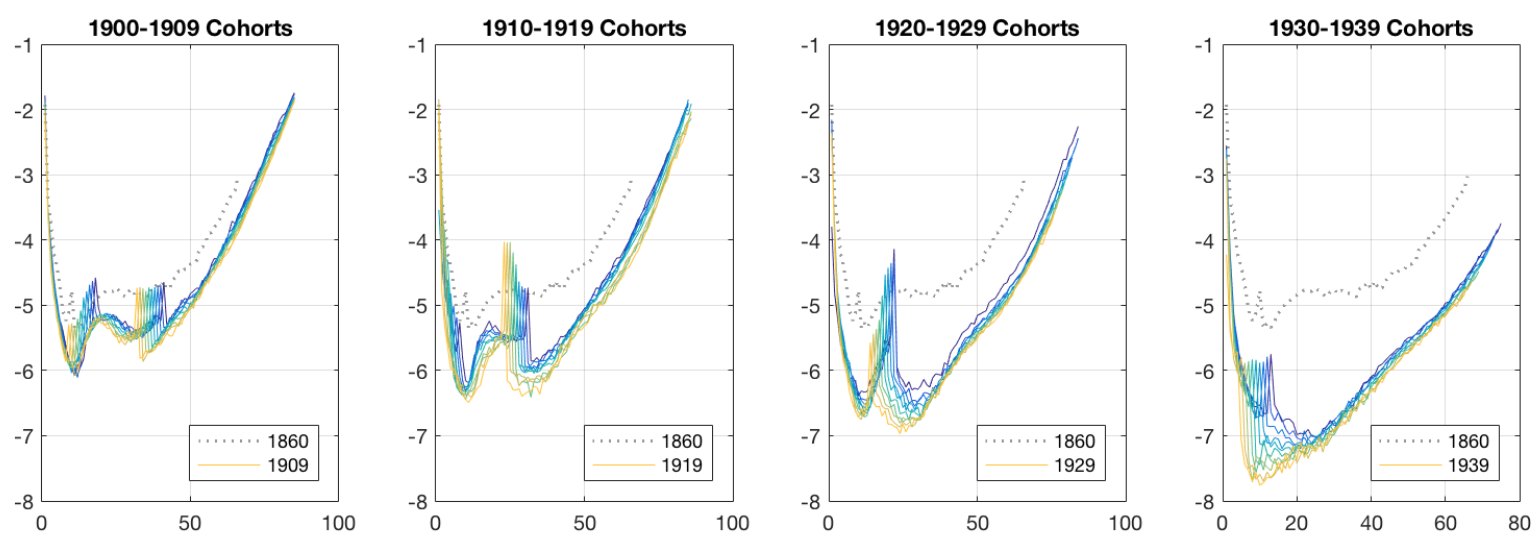

Note: Human Mortality Data 
Figure 16: Age profile of mortality of men born in France between 1860 and 1940, by decade Log-Mortality Rates. French Men cohorts bom between 1860 and 1939 (Source:HMD)
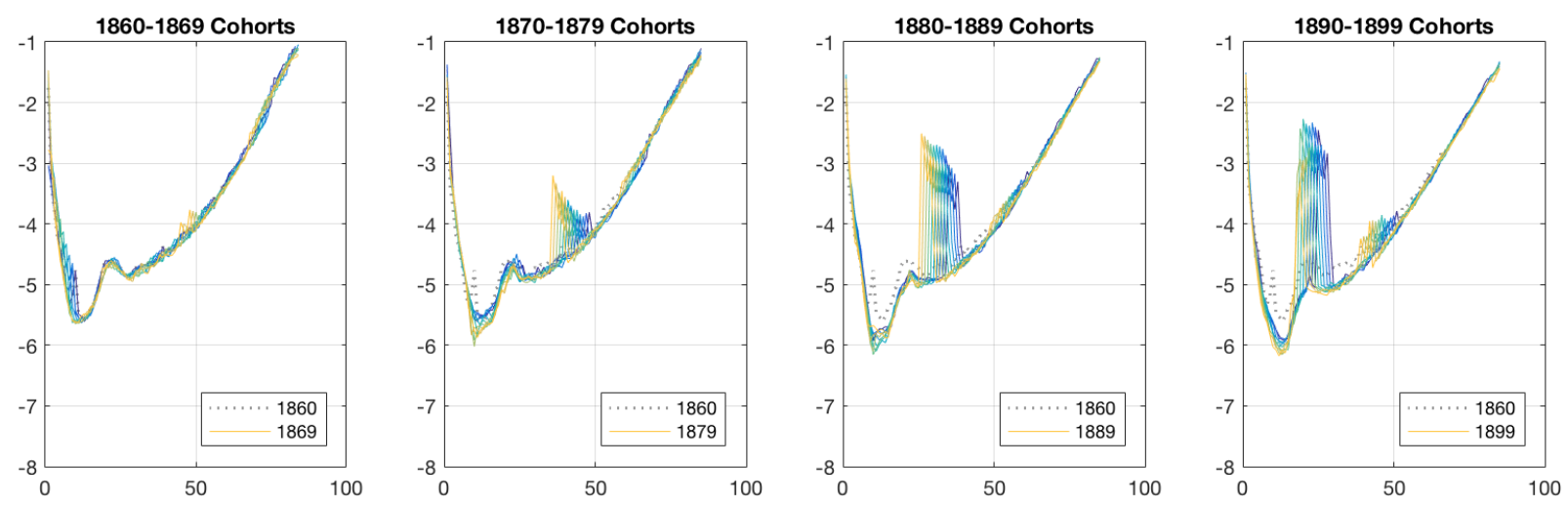

용
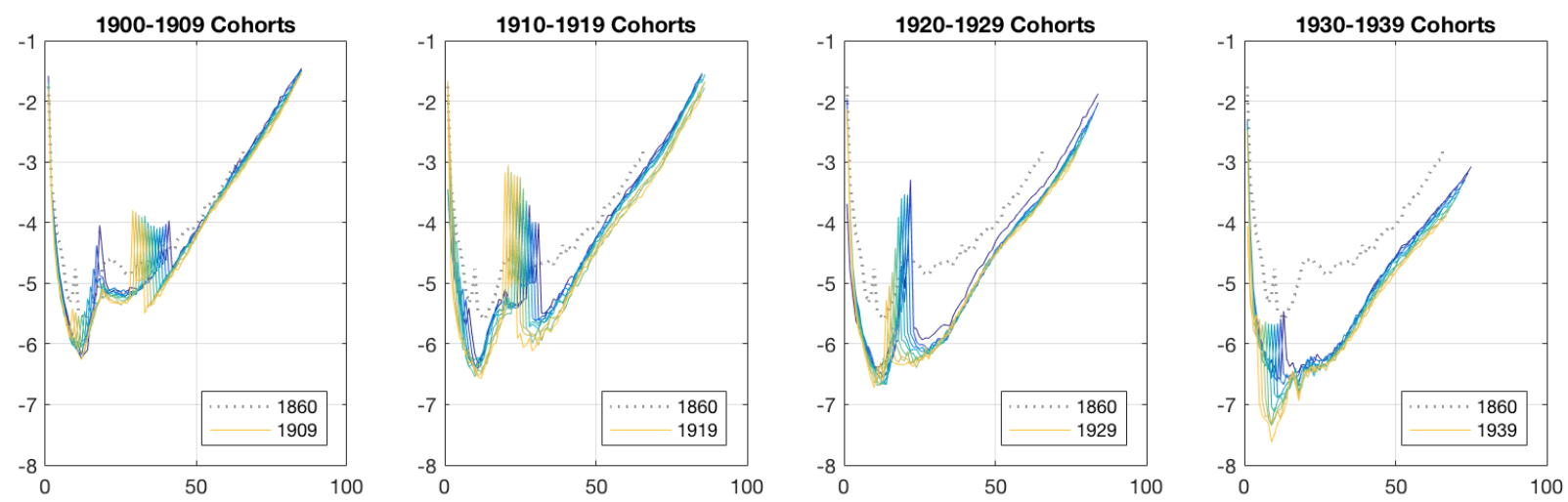

Note: Human Mortality Data 
Figure 17: Model fit for humans and primates

(a) French men born in 1816

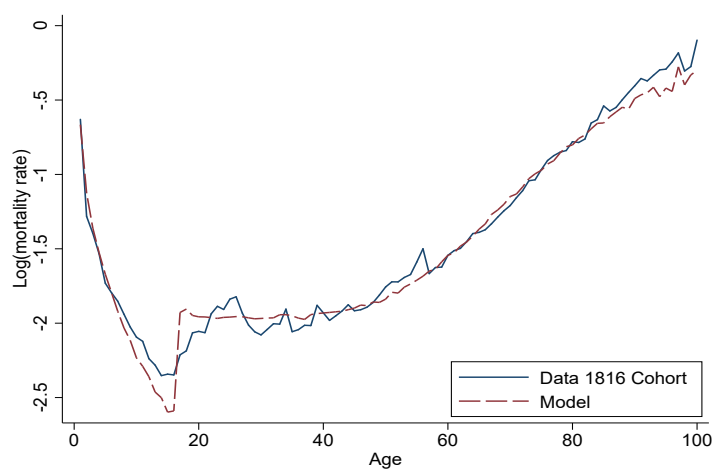

(b) Male chimpanzees

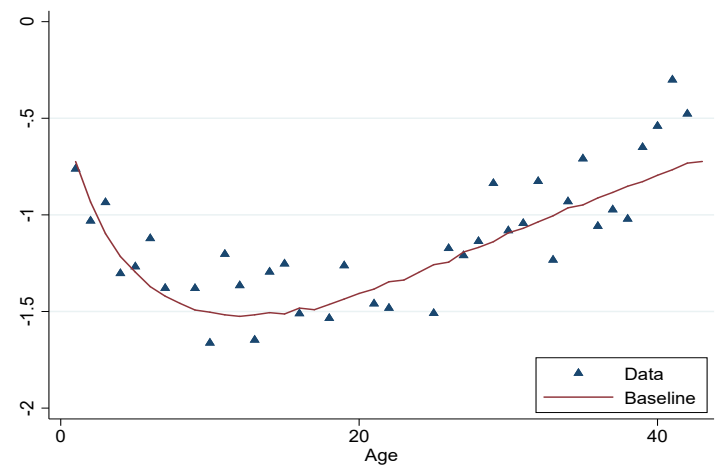

Note: Panel $a$ shows the data and estimated curve for French men born in 1816. Panel $b$ shows the data and estimated parameters for male chimps. Appendix Tables 1 and 2 show the estimated parameters. 
Figure 18: Model fit for birth cohorts born 1816-1923

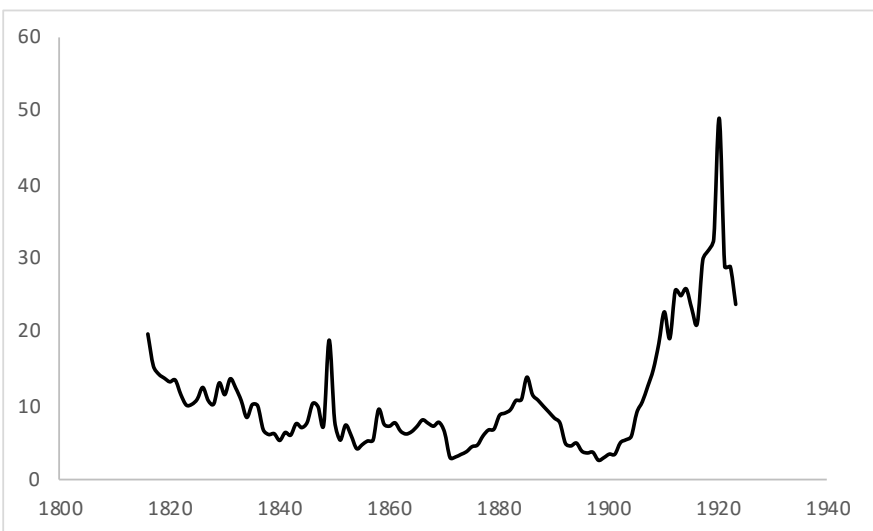

Note: this figure shows the fit of the model for each birth cohort. The fit is measured as the sum of quadratic errors between the estimated survival curve and the data at each age, defined as $\sum_{a}\left(\hat{S}_{a}-S_{a}\right)^{2}$. 
Figure 19: Increasing the lifetime depreciation rate by $50 \%$ by age
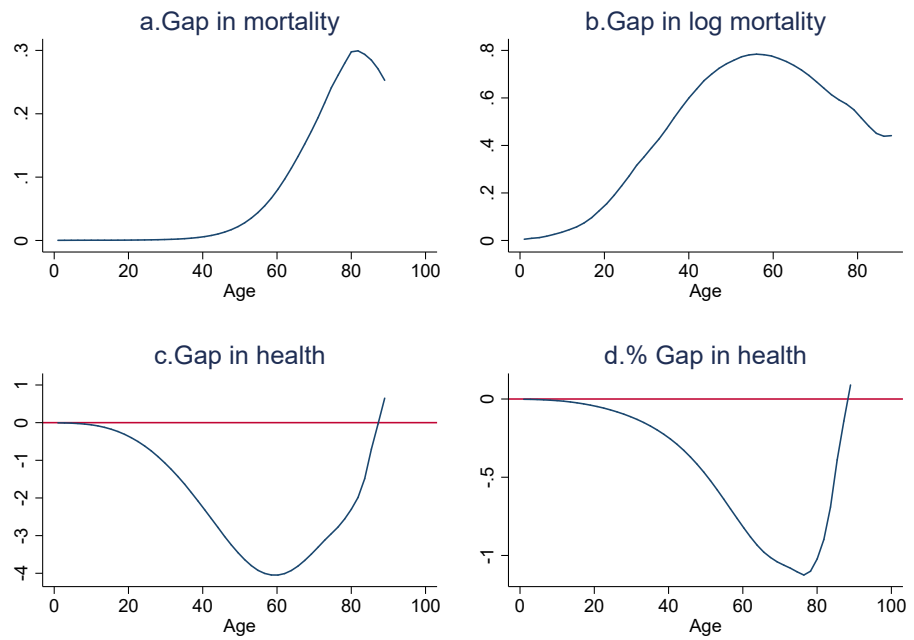

Note: The Figure shows the gap in mortality or health between a baseline population and a population with a $50 \%$ higher depreciation rate $\delta$. Gap is computed as MR(low)-MR(high), or H(low)$\mathrm{H}$ (high). The figures become very noisy after age 90 because there are almost no survivors, so we do not include these data points. Simulated data for two population of 500,000 individuals each. The baseline parameters are the same as in Figure 3b. 
Figure 20: Effect of exogenous temporary shocks at age 20

(a) Gaps in mortality
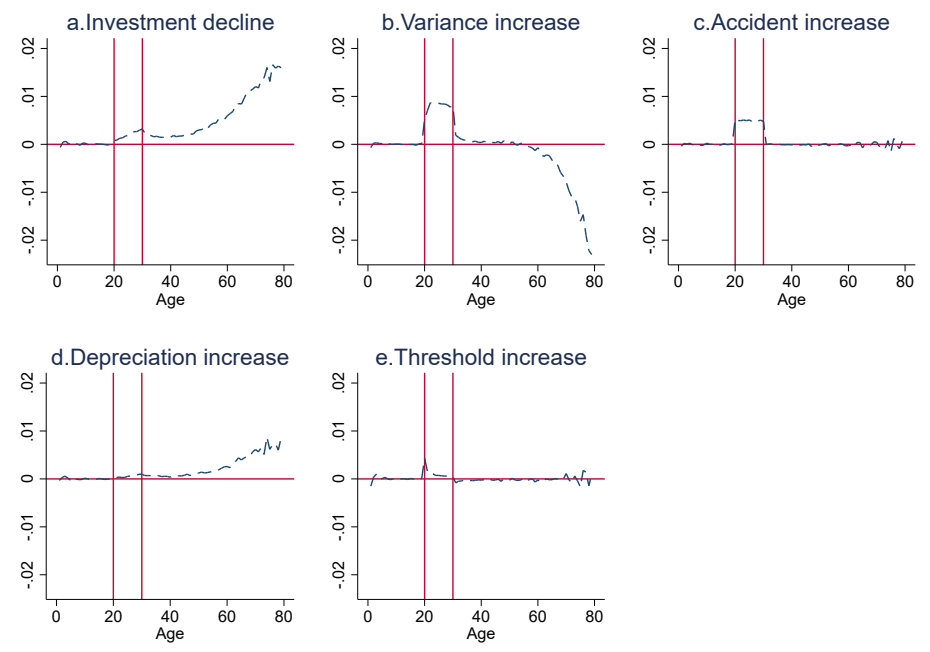

(b) Log gaps in mortality
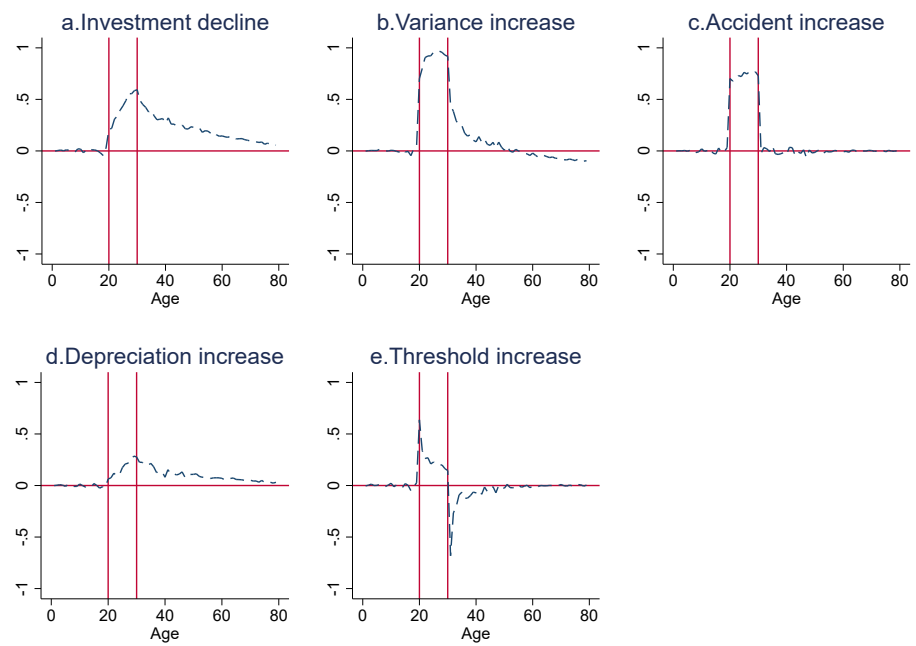

Note: The figure shows the effects of a temporary change in a single parameter occuring at age 20. The shock lasts for 10 years, ending at age 30. Each figure shows the different in mortality that results from a temporary shock, relative to the counterfactual of no shock. In essence these figures plot the pattern that would be predicted in an event study, where the coefficient of a dummy for the affected population is intereacted with time fixed effects. Panel a shows the gaps in levels and panel $b$ shows the gaps in logs. The baseline parameters are the same as in Figure 3b. 
Table 1: Modeling prime-age mortality. French Cohort born in 1816

\begin{tabular}{|c|c|c|c|c|c|}
\hline & & $(1)$ & $(2)$ & (3) & $(4)$ \\
\hline Gender & & \multicolumn{2}{|r|}{ Females } & \multicolumn{2}{|r|}{ Males } \\
\hline Model & & Baseline & With adolescent hump & Baseline & With adolescent hump \\
\hline Initial mean health & $\mu_{H}$ & 0.9115 & 0.8634 & 0.7091 & 0.7104 \\
\hline Investment & $I$ & 0.1336 & 0.4075 & 0.1209 & 0.4432 \\
\hline Standard Deviation of Shock & $\sigma_{e}$ & 0.5556 & 1.0241 & 0.4700 & 1.0785 \\
\hline Depreciation & $\delta$ & 0.0010 & 0.0006 & 0.0014 & 0.0004 \\
\hline Aging & $\alpha$ & 1.4350 & 1.7849 & 1.3182 & 1.8883 \\
\hline Adolescent Hump* & $\kappa$ & & 0.0086 & & 0.0097 \\
\hline Fit (survival curve) ${ }^{\wedge}$ & & 155.06 & 12.36 & 143.07 & 16.38 \\
\hline Eit $\left(\log\right.$ of $\left.q_{x}\right)$ & & 3.01 & 0.74 & 4.02 & 0.91 \\
\hline Fit (death distribution)** & & 6.21 & 3.35 & 18.10 & 96.16 \\
\hline Actual Life Expectancy & & 38.25 & 38.25 & 35.93 & 35.93 \\
\hline Predicted Life Expectancy & & 38.43 & 38.28 & 36.13 & 35.94 \\
\hline Counterfactual Life expectancy^^^ & & & 45.86 & & 43.54 \\
\hline
\end{tabular}

In this table we fit the model to the 1816 population with and without an exogenous increase in the accident rate occurring in adolescence.

*The estimate in this row corresponds to the value of the parameter $\kappa$ after the onset of adolescence. Adolescence starts at age $=(-0.0175 x$ calendar year $)+47.4$ for all women, based on the estimates provided in de La Rochebrochard (2000). Adolescence starts one year later for men.

$\wedge$ Our main fit criteria is the sum of squared errors of the survival rate at each age. We also report the fit as the sum of squared errors of the $\log$ of $q_{x}$ (the probability of dying between ages $x$ and $x+1$ ) and the distribution of deaths. We don't target these moments directly-we target the survival curve.

**To make the fit of the age distribution comparable across columns we use the (normalized) number of deaths as weights.

$\wedge \wedge$ Counterfactual Life Expectancy is computed by holding all estimated parameters fixed and setting the adolescent hump to 0 . 
Table 2: Estimated parameters for chimpanzees living in the wild

\begin{tabular}{|c|c|c|c|c|c|c|c|}
\hline & & (1) & (2) & (3) & (4) & (5) & (6) \\
\hline Gender & & & Female & & & Male & \\
\hline Model & & Basic model & With hump at 8 & With hump at 14 & Basic model & With hump at 8 & With hum \\
\hline Initial mean health & $\mu_{H}$ & 0.9995 & 1.0337 & 1.0266 & 1.8421 & 3.6402 & 4.533 \\
\hline Investment (annual) & $I$ & 0.3488 & 0.3613 & 0.3603 & 0.7646 & 1.1364 & 0.73 \\
\hline Standard Deviation of Shock & $\sigma_{e}$ & 1.1299 & 1.1806 & 1.1787 & 2.7145 & 4.9725 & $5.0^{\prime}$ \\
\hline Depreciation & $\delta$ & 0.0598 & 0.0600 & 0.0593 & 0.0620 & 0.0510 & 0.00 \\
\hline Aging & $\alpha$ & 0.7627 & 0.7736 & 0.7763 & 1.0319 & 1.2501 & 1.802 \\
\hline Adolescent Hump* & $\kappa$ & & 0.000040 & 0.000002 & & 0.000378 & 0.0000 \\
\hline \# of individuals at birth & & 144 & 144 & 144 & 122 & 122 & 12 \\
\hline \# of moments reported & & 55 & 55 & 55 & 43 & 43 & \\
\hline Fit (survival curve) $)^{b}$ & & 111.12 & 110.20 & 110.25 & 128.43 & 99.26 & 65.6 \\
\hline Fit $\left(\log\right.$ of $\left.q_{x}\right)$ & & 2.08 & 2.07 & 2.09 & 1.21 & 1.14 & 1.06 \\
\hline Actual Life Expectancy & & $15.38(13.4)^{a}$ & $15.38(13.4)^{a}$ & $15.38(13.4)^{a}$ & 14.47 & 14.47 & 14.4 \\
\hline Predicted Life Expectancy & & 15.35 & 15.35 & 15.35 & 14.52 & 14.50 & 14. \\
\hline
\end{tabular}

Columns (1) and (4) estimate the model without an adolescent hump. Columns (2) and (4) estimate the model with an exogenous increase in accidents in adolescence at age 8. Columns (3) and (6) estimate the model with an exogenous increase in accidents in adolescence at age 14. Because the data are noisy the second/third model is not a substantially better fit than the first. All are however excellent fits for this population.

Data sources: Life tables for primates in the wild come from Bronikowski et al. (2011). In the wild population data come from Brazil, Costa Rica, Kenya, Tanzania, Madagascar and Rwanda.

a. Life expectancy in parenthesis corresponds to the one reported in Bronikowski et al. (2011).

$b$. We target the survival curve and compute the sum of squared errors - the data provided are in the form of survival rates.

*Adolescence starts at age 8 . 
Table 3: Robustness checks for 1816

\begin{tabular}{|c|c|c|c|c|c|c|c|c|}
\hline & & $(1)$ & $(2)$ & (3) & $(4)$ & (5) & (6) & $(7)$ \\
\hline & & Basic & $\kappa_{b}$ & $\kappa_{b}$ at $T \sim N()$ & $T \sim N()$ estimated & Weight & Target death & Truncation at 90 \\
\hline Initial mean health & $\mu_{H}$ & 0.8634 & 0.8917 & 0.8635 & 0.7503 & 0.8020 & 0.7327 & 0.8784 \\
\hline Investment & $I$ & 0.4075 & 0.4322 & 0.4149 & 0.3712 & 0.4180 & 0.4743 & 0.4200 \\
\hline Standard Deviation of Shock & $\sigma_{e}$ & 1.0241 & 1.0713 & 1.0367 & 0.8369 & 0.9971 & 0.9930 & 1.0552 \\
\hline Depreciation & $\delta$ & 0.0006 & 0.0005 & 0.0005 & 0.0005 & 0.0006 & 0.0006 & 0.0006 \\
\hline Aging & $\alpha$ & 1.7849 & 1.8321 & 1.8153 & 1.8011 & 1.7807 & 1.7950 & 1.7973 \\
\hline Adolescent Hump* & $\kappa_{a}$ & 0.0086 & 0.0089 & 0.0089 & 0.0112 & 0.0093 & 0.0108 & 0.0087 \\
\hline Accident rate before adolescence & $\kappa_{b}$ & & 0.0005 & & & & & \\
\hline Mean* & & & & 15.6 & 14.29 & & & \\
\hline Standard deviation* & & & & 1.32 & 15.71 & & & \\
\hline Fit (survival curve) ${ }^{\wedge}$ & & 12.36 & 13.03 & 11.49 & 3.75 & 15.86 & 173.74 & 12.36 \\
\hline Fit $\left(\log\right.$ of $\left.q_{x}\right)$ & & 0.74 & 0.65 & 0.57 & 0.40 & 0.92 & 1.93 & 0.56 \\
\hline Fit (death distribution)** & & 3.35 & 39.55 & 21.82 & 2.25 & 6.89 & 4.48 & 28.17 \\
\hline Actual Life Expectancy & & & & & 38.25 & & & \\
\hline Predicted Life Expectancy & & 38.28 & 38.29 & 38.28 & 38.26 & 38.33 & 39.27 & 38.27 \\
\hline Counterfactual Life expectancy^^${ }^{\wedge \wedge}$ & & 45.86 & 46.45 & 46.08 & 48.82 & 46.66 & 49.49 & 45.86 \\
\hline
\end{tabular}

${ }^{*}$ Adolescence starts at age $=(-0.0175 \times$ calendar year $)+47.4$ in columns $1,2,5,6$ and 7.

In column 3 the timing of adolescence is assumed to follow a normal distribution with mean value (- $0.0175 \times$ calendar year) + 47.4, and standard deviation 1.3285, calculated from the table of 1975 girls in de La Rochebrochard (2000). In column 4 we estimate the mean and the standard deviation of the onset of adolescence.

In column 5 we investigate what happens if we use the (normalized) number of deaths as weights in the estimation. In column 6 we use weights and target the distribution of the ages at death instead of the survival curve. In the column 7 we use only data up to age 90 to see what the effect of censoring is and because the data after 90 are estimated. The estimates are somewhat sensitive to these choices. The predicted life expectancy is very close in all cases (the error in the predicted life expectancy is less then 0.1 years of life), except when we target the age at death distribution (the prediction is off by about a year). But the counterfactual predictions are sensitive: eliminating the hump results in a loss of life of 7.58 years on the baseline model and 9.81 in the worse model.

$\wedge$ Our main fit criteria is the sum of squared errors of the survival rate at each age. We also report the fit as the sum of squared errors of the $\log$ of $q_{x}$ (the probability of dying between ages $x$ and $x+1$ ) and the distribution of deaths. We don't target these moments directly-we target the survival curve.

$\wedge \wedge$ Counterfactual Life Expectancy is computed by holding all estimated parameters fixed and setting the adolescent hump to 0.

${ }^{*}$ To make the fit of the age distribution comparable across columns we use the (normalized) number of deaths as weights.

In column 4 we target the survival curve but use the (normalized) number of deaths as weights

In column 5 we target the distribution of the age at death and we use the number of deaths as weights. 
Table 4: Estimated parameters for French Women and Men for Selected Cohorts

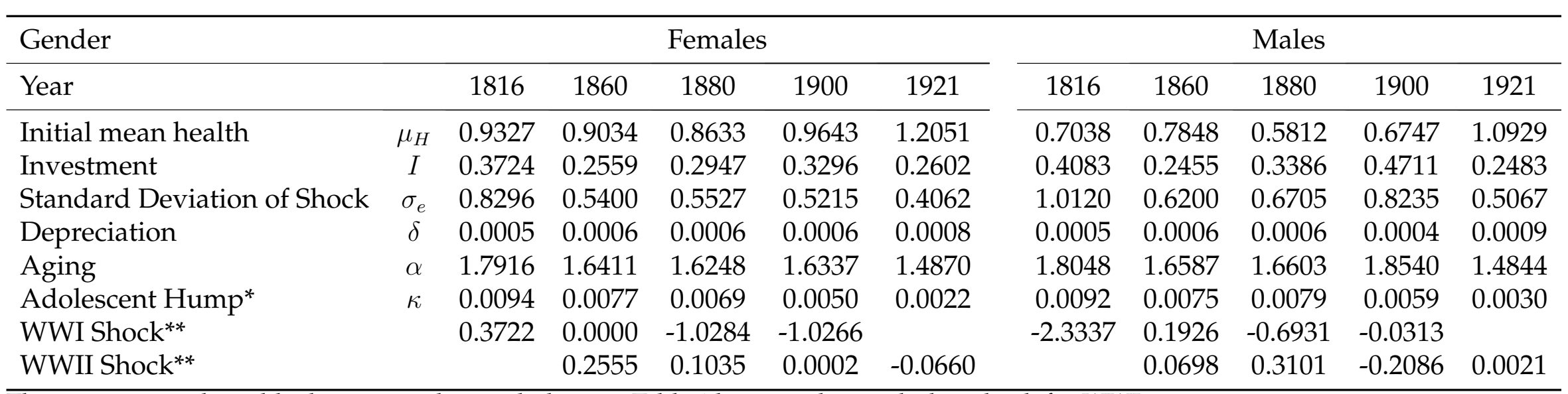

The estimates in this table do not exactly match those in Table 1 because they include a shock for WWI.

*The estimate in this row corresponds to the value of the parameter $\kappa$ after the onset of adolescence. Adolescence starts at age $=(-0.0175 x$ calendar year $)+47.4$ for all women, based on the estimates provided in de La Rochebrochard (2000). Adolescence starts one year later for men.

**The estimates in this row corresponds to the value of the parameter during the world wars. For example the column for the 1900 female cohort shows that $I$ was about 0.96 throughout life but decreased to -1.02 during WWI and to 0.005 during WWII. 
Table 5: Estimated parameters for World Wars for French Men born in 1896

\begin{tabular}{|c|c|c|c|}
\hline & & (1) & (2) \\
\hline Initial condition & $\mu_{H}$ & 1.1417 & 0.8448 \\
\hline Investment & $I$ & 0.4548 & 0.3009 \\
\hline Standard Deviation of Shock & $\sigma_{e}$ & 1.0259 & 0.5983 \\
\hline Depreciation & $\delta$ & 0.0002 & 0.0005 \\
\hline Aging & $\alpha$ & 2.0052 & 1.6913 \\
\hline Adolescence Hump* & $\kappa$ & 0.0025 & 0.0037 \\
\hline WWI Shock** & & -1.3104 & \\
\hline Shock in 1914 & & & -2.9302 \\
\hline Shock in 1915 & & & -0.7485 \\
\hline Shock in 1916 & & & -0.5333 \\
\hline Shock in 1917 & & & 0.2570 \\
\hline Shock in 1918 & & & -0.1191 \\
\hline WWII Shock** & & 0.0577 & 0.1560 \\
\hline Fit (survival curve)^ & & 218.64 & 11.57 \\
\hline Fit $\left(\log\right.$ of $\left.q_{x}\right)$ & & 2.65 & 1.27 \\
\hline Fit during WWI $\left(\log\right.$ of $\left.q_{x}\right) \sim$ & & 1.09 & 0.09 \\
\hline$\%$ Difference in \# deaths during WWI & & -0.14 & -0.05 \\
\hline Fit during WWII $\left(\log\right.$ of $\left.q_{x}\right) \sim$ & & 0.10 & 0.09 \\
\hline$\%$ Difference in \# deaths during WWII & & 0.02 & -0.11 \\
\hline Actual Life Expectancy & & \multicolumn{2}{|c|}{37.94} \\
\hline Predicted Life Expectancy & & 37.98 & 37.96 \\
\hline Counterfactual Life expectancy without WWI^^ & & 54.13 & 54.74 \\
\hline Counterfactual Life expectancy without WWII^^^ & & 39.90 & 39.10 \\
\hline Counterfactual Life expectancy ${ }^{\wedge \wedge}$ & & 56.22 & 55.97 \\
\hline
\end{tabular}

*Hump is modeled as a accident rate that starts in adolescence, set to happen at (- 0.0175

* calendar year) + 47.4 + 1based on the estimates provided in de La Rochebrochard (2000) and the assumption that adolescence starts one year later for men.

${ }^{* *}$ The estimates in this row corresponds to the value of the parameter during the world wars. For example the first column shows that Iwas about 1.1417 throughout life but decreased to -1.3104 during WWI and decreased to 0.0577 during WWII. The same applies to column (2). In column 2, we allow the shocks in investment to vary across years during WWI.

$\wedge^{\wedge}$ Our main fit criteria is the sum of squared errors of the survival rate at each age. We also report the fit as the sum of squared errors of the $\log$ of $q_{x}$ (the probability of dying between ages $x$ and $x+1$ ). We don't target these moments directly-we target the survival curve.

$\wedge^{\wedge}$ Counterfactual Life Expectancy is computed by holding all estimated parameters fixed and setting the war parameters to the parameter $I$.

$\sim$ This is computed as sum of squared errors during the war years. A lower number is better.

$\sim \sim$ This is computed as (predicted - actual)/actual

To make the fit of the age distribution comparable across columns we use the (normalized) number of deaths as weights. 


\section{Appendix B: Everyone dies}

The model is defined as follows:

$$
\left\{\begin{array}{l}
H_{a}=H_{a-1}-d(a)+I+\varepsilon_{t} \quad \text { if } D_{a-1}=0 \\
D_{a}=\mathbb{I}\left(H_{a} \leq \underline{H}, D_{a-1}=0\right), \\
D_{0}=0
\end{array}\right.
$$

with $d(a)=\delta \cdot a^{\alpha} \delta \in(0, \infty), \alpha \in(0, \infty)$, and $I \in \mathbb{R} . \underline{H}$ and $\sigma_{H}^{2}$ are normalized to be 0 and 1 , respectively. Let $\hat{H}_{a} \equiv \mathbb{E}\left[H_{a} \mid H_{a}>0\right]$ denote the average health in the living population with age $a$ and $\sigma_{\hat{H}_{t}} \equiv \operatorname{Var}\left[H_{a} \mid H_{a}>0\right]$ the variance of health among the living.

\section{Proposition 1. Everyone dies eventually.}

The cumulative distribution function of our process can be bounded above by a process easier to study. Consider the process $\left\{H_{a}^{*}\right\}_{a=1}^{\infty}$, defined by $H_{0}^{*}=H_{0} \sim$ $\mathcal{N}\left(\mu_{H}, \sigma_{H}^{2}\right)$ and the recurrence relation:

$$
H_{a}^{*}=H_{a-1}^{*}+I-\delta \cdot a^{\alpha}+\varepsilon_{a}, \varepsilon_{a} \sim \mathcal{N}\left(0, \sigma_{\varepsilon}^{2}\right)
$$

The process is similar to the one in our model except that there is no truncation. It is easy to tell that $0 \leq P\left(H_{a}>z\right) \leq P\left(H_{a}^{*}>z\right)$ for any $z>0$. Now for any $a \geq 0$, $H_{t}^{*}$ is normally distributed with mean

$$
\mu_{H_{a}^{*}}=\mu_{H}+I \cdot a-\delta \sum_{k=1}^{a} k^{\alpha}
$$

and standard deviation

$$
\sigma_{H_{a}^{*}}=\sqrt{\sigma_{H}^{2}+a \cdot \sigma_{\varepsilon}^{2}}
$$

Hence, $P\left(H_{a}^{*}>z\right)=1-\Phi\left(\frac{z-\mu_{H_{a}^{*}}}{\sigma_{H_{a}^{*}}}\right)$, where $\Phi$ is the CDF of the standard normal distribution. As $a \rightarrow \infty$, we have $\mu_{H_{a}^{*}} \sim I \cdot a-\delta \cdot \frac{a^{\alpha+1}}{\alpha+1}$ and $\sigma_{H_{a}^{*}} \sim \sqrt{a} \cdot \sigma_{\varepsilon}$. Therefore if $\alpha>0, \frac{\mu_{H_{a}^{*}}}{\sigma_{H_{a}^{*}}} \rightarrow-\infty$ as $a \rightarrow \infty$.

Remark: Extended model with Accident shocks Proposition 1, 2 and 3 hold for the extended model with accident shocks drawn indepently from the health 
status. Because accident shocks are drawn independently from the health status, they leave the $c d f$ of health unchanged and therefore the proofs are unaffected. 


\section{Appendix C: Notes on the empirical method}

\section{Data}

Territory changes. The table below describes the details of the changes in territory that took place in France since 1816.

\begin{tabular}{ll}
\hline Year & Territorial Changes \\
\hline \hline 1861 & Annexion of departements of Savoie and Haute-Savoie, and of Comte de Nice \\
1869 & Franco-Prussian war: loss of Alsace-Lorraine \\
$1914-$ & WWI: East of France, from Nord Pas-de-Calais to Vosges, is occupied by German military. \\
1919 & At the end of WWI, Alsace-Lorraine is re-integrated to French territory \\
1939 & WW2: Loss of Alsace-Lorraine \\
1943 & WW2: Loss of Corsica \\
1945 & Current territory: Alsace-Lorraine and Corsica are re-integrated to French territory
\end{tabular}

These changes in territory results in large changes in the population and death counts. This is illustrated below for population. It is unclear how to compute mortality in the year of the change. We compute it by using a weighted average of the population at the beginning and end of the year.

Migration. In the HMD cohort population counts are available. However, because of migrations, these counts cannot be used to derive a survival curve for a cohort. Because of net positive immigration occurring in France, the number of individuals in a given cohort can even increase from one year to the next. This is especially true at the end of the Algerian War. (e.g. the size of the female cohort born in 1910 increases from 300, 369 to 303,273 between 1962 and 1963, despite a reported mortality rate of 0.5162 . The unit of analysis in our model of mortality is a country cohort, hence abstracts from migration. In our model the mortality rates coincide exactly with the slope of the survival curve. This is not true in the HMD. The population of the cohort melts natives and immigrants of the same age.

\section{Computing the death rates, survival rates and life expectancy}

Death rates. When taking our model to the data we target the most direct counterpart of our modeled cohort "mortality rate", which is computed as the number of individuals who died during a year, divided by the number of individuals alive at the beginning of the day. In typical life tables this number corresponds to what 
demographer call $q_{t}$, the probability of dying in a given year, and is conceptually distinct to the mortality rate, denoted by $m_{t}$. The main difference lies in adjusting the denominator - the size of the population. As more individuals die during the year the population needs to be adjusted to estimate the size of the remaining population exposed to the risk of death. Because our baseline model does not take this adjustment into account, we compute a direct counterpart of our theoretical object. Therefore, we compute the raw death rate in year $t$ for a given cohort, $q_{t}$, as follows:

$$
q_{t}=\frac{D_{t}}{N_{t}}
$$

where $D_{t}$ is the death count for year $t$ from the HMD cohort table and $N_{t}$ is the population on January 1st of year $t$. The HMD makes adjustments to compute a probability that is corrected for the fact that the data do not tract the same individuals over time, so the probability of dying is not correctly computed for a given cohort. The $q$ we estimate with the raw counts is very similar to what is reported by the HMD except for the first year of life and the last years of life as shown in Figure 13. This results in our under-estimating life expectancy somewhat.

Survival curves. We compute the survival curve recursively as follows. After initializing $S_{0}=100$, we iteratively compute:

$$
S_{t}=S_{t-1} \times\left(1-q_{t-1}\right)
$$

Life expectancy. Life Expectancy (LE) is an important statistics for the health profile of a given cohort. We compute LE as a way of comparing our model to the data in a parsimonious way. While we try to provide informative estimates of cohort life expectancy, we do not claim that their accuracy is comparable to demographic studies. Nevertheless, as we treat the series generated by our model in exactly the same manner as the data series, we obtain pairs of LE that are readily comparable.

\section{Estimation routine}

We compute our estimates using Matlab's canned fminsearch routine, a downhill simplex method, and Powell (1964)'s conjugate direction method. We first estimate the model using fminsearch until the objective function changes by less than $10^{-3}$. 
The objective function is the sum of squared errors between the model's survival's curve and the one from the data. We then use these estimates as starting values for Powell's routine. Once Powell's routine converges, we use the estimated values from this procedure and implement fminsearch again until it converges. The total estimations on the UCLA computing cluster takes several hours. We experimented with different initial values for the parameters. The reported estimates correspond to the lowest final function value.

\section{Bootstrapping standard errors}

Estimates from sample data come with standard errors. However, the mortality rates in the HMD are computed from birth certificates of the total population, not a sample of it. A typical cohort in our study counts 400,000 individuals. As a result, the standard errors are negligible and all of the parameter uncertainty comes from model mispecification and data inaccuracy rather than sampling variation. We therefore do not report standard errors for the French cohorts.

In contrast, we do compute the standard errors for the chimpanzee estimates as the data in that case consist of samples of one or two hundreds of individuals. One way of bootstrapping the standard errors, given a series of mortality rates for a cohort, is to view each sample of size $\mathrm{N}$ as a sequence of Bernoulli trials with varying success rates. Alternatively, one can view the survival curve of a population of size $N$ as an $N \times 1$ vector of age at death. One can produce bootstrap estimates by drawing with replacement $M$ subsamples of size $N$ and compute the empirical survival curve. 